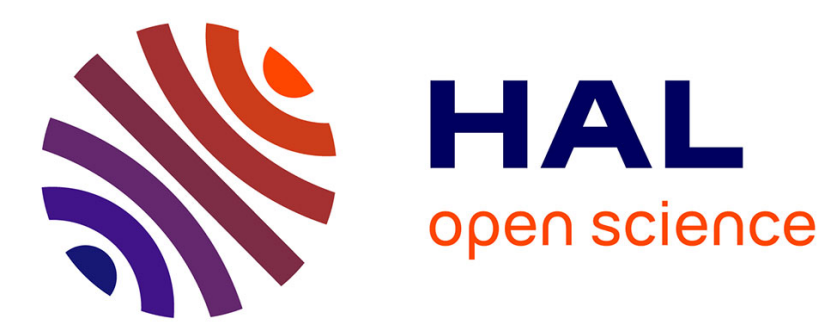

\title{
Modèle d'anisotropie des propriétés magnétiques induites par l'orientation préférentielle de forme dans une roche déformée
}

\author{
R. Vergne, P. Mollard, P. Vialon, P. Rochette
}

\section{- To cite this version:}

R. Vergne, P. Mollard, P. Vialon, P. Rochette. Modèle d'anisotropie des propriétés magnétiques induites par l'orientation préférentielle de forme dans une roche déformée. Revue de Physique Appliquée, 1988, 23 (5), pp.891-918. 10.1051/rphysap:01988002305089100 . jpa-00245892

HAL Id: jpa-00245892

https://hal.science/jpa-00245892

Submitted on 1 Jan 1988

HAL is a multi-disciplinary open access archive for the deposit and dissemination of scientific research documents, whether they are published or not. The documents may come from teaching and research institutions in France or abroad, or from public or private research centers.
L'archive ouverte pluridisciplinaire HAL, est destinée au dépôt et à la diffusion de documents scientifiques de niveau recherche, publiés ou non, émanant des établissements d'enseignement et de recherche français ou étrangers, des laboratoires publics ou privés. 


\title{
Modèle d'anisotropie des propriétés magnétiques induites par l'orientation préférentielle de forme dans une roche déformée
}

\author{
R. Vergne ( $\left.{ }^{(}\right)$, P. Mollard ( $\left.{ }^{1}\right)$, P. Vialon $\left({ }^{2}\right)$ et P. Rochette $\left({ }^{1,2}\right)$ \\ (1) Laboratoire Louis Neel, CNRS-USTMG, $166 \mathrm{X}, 38042$ Grenoble Cedex, France \\ (2) Laboratoire de·Géophysique Interne et de Tectonophysique, IRIGM, BP 68, 38042 Saint-Martin-d'Hères \\ Cedex, France
}

(Reçu le 29 septembre 1987, révisé le 22 décembre 1987, accepté le 29 janvier 1988)

\begin{abstract}
Résumé. - Le but essentiel de ce travail est de montrer comment il est possible, à l'aide d'arguments simples : de justifier les corrélations observées entre l'anisotropie des propriétés magnétiques d'une roche et sa déformation interne et de les chiffrer dans le cas particulier de l'O.P.F. Pour modéliser une roche polyminérale, on considère l'ensemble des grains d'un même minéral : les marqueurs et le reste de la roche : la matrice. On applique à cet ensemble le modèle hydrodynamique en se bornant au cas d'une déformation coaxiale orthorhombique isovolumique et en négligeant les interactions entre grains. La déformation finie s'exprime simplement par une transformation linéaire dans un système d'axes invariants au cours de celle-ci : les axes principaux de déformation. Le mouvement des marqueurs et l'invariance de leur nombre conduit à l'existence d'une densité d'orientation de leurs axes, définie dans le même repère sur la sphère de rayon unité. Son expression est celle qu'en a donné Fernandez (1984). Du point de vue magnétique, le modèle privilégie les processus d'aimantation. Leur choix résulte d'une analyse basée sur la morphologie et la structure magnétique des constituants possibles d'une roche polyminérale et des divers mécanismes d'aimantation correspondants. On peut ainsi préciser certaines situations modélisables et déterminer les grandeurs magnétiques qui les caractérisent. Nous avons retenu pour les marqueurs un processus de grain fin, un processus de polydomaine, et une aimantation décrite par une loi de Rayleigh. Le calcul de la grandeur considérée s'effectue en supposant la matrice non magnétique et en négligeant les interactions entre grains. Le mécanisme de grain fin conduit à l'étude de l'aimantation rémanente (ARIS, ATR). Il définit un milieu non linéaire doué de mémoire dans lequel l'aimantation rémanente dépend de l'histoire magnétique et tectonique de la roche. Pour illustrer ce fait, nous avons calculé l'aimantation rémanente résultant de la déformation d'un état isotrope initialement aimanté (ARISD, ATRD). Pour les polydomaines, nous avons évalué la susceptibilité en champ faible. Dans le cas de grains suivant une loi de Rayleigh, on détermine l'ensemble des grandeurs : susceptibilité réversible et irréversible, aimantation rémanente, aimantation. Nous avons étudié en détail le cas le plus simple que l'on puisse envisager : la déformation est un aplatissement ou étirement de révolution et les marqueurs sont des grains fins ou des polydomaines; ce qui représente les deux extrêmes d'une granulométrie étendue. Cette étude montre : 1) que l'on peut établir une relation quantitative entre l'anisotropie de la déformée et celle des grandeurs magnétiques pertinentes ; 2) que dans une certaine mesure, l'information obtenue est du même ordre quelle que soit la grandeur considérée ; ce qui souligne la cohérence du modèle. Ce dernier ne se ramène pas à une transformation linéaire et les grandeurs ne sont pas en général décrites par un tenseur de rang deux. Une analyse plus fine des résultats permet de chiffrer dans le cadre envisagé la similitude entre fabriques mécanique et magnétique et l'approximation que l'on fait en assimilant les grandeurs à un tel tenseur.
\end{abstract}

Abstract. - This work is essentially aimed at showing how one can, with the help of simple arguments, justify the correlations observed between the anisotropy of the magnetic properties of a rock and its internal deformation, and quantify them in the particular case of the preferential shape orientation mechanism. A polymineral rock is pictured by a population of grains of a given mineral : the so-called markers, and the remainder of the rock : the matrix. The hydrodynamical model is applied to this population in the special case of coaxial, orthorhombic, isovolumic deformation, by neglecting grain interactions. The finite deformation is simply depicted by a linear transformation in a system of invariant coordinate axes which are the principal axes of deformation. The motion of markers, together with the invariance of their number, leads to an angular distribution of their axes defined in the same reference frame on the sphere of unit radius, which was derived 
by Fernandez (1984). From a magnetic standpoint, the model focuses on magnetization processes selected from an analysis based on the morphology and magnetic structure of the constituents in a polymineral rock, and the various corresponding magnetization mechanisms. It is thus possible to precise some situations to which the model can apply, and to determine the corresponding magnetic quantities. We focus on the following processes for the magnetic markers : single-domain particles, polydomains, and magnetization obeying a Rayleigh law. The calculation of magnetic variables is performed by assuming a non-magnetic matrix and negligible grain interactions. The single-domain mechanism leads to studying the remanent magnetisation (SIRM, TRM). It enables us to define a non-linear medium with a memory, in which the remanent magnetization depends on both the magnetic and tectonic histories of the rock. We illustrate this concept by calculating the remanent magnetization arising from the deformation of an initially magnetized isotropic state (DSIRM, DTRM). For polydomains, we determine the weak-field susceptibility. For grains obeying a Rayleigh law, we determine the reversible and irreversible susceptibilities, magnetization and remanent magnetization. We work out in detail the simplest possible case in which the deformation consists of a revolution flattening or stretching, and the markers are single-domain or polydomain particles, corresponding to the extreme situations encountered in a broad granulometry. This study shows that : 1) one can establish a quantitative relationship between the anisotropy of the deformation and that of the relevant magnetic quantities ; 2) to some extent, the information obtained is the same, whichever the quantity considered, which confirms the coherence of the model. The latter cannot be reduced to a linear transformation, and the magnetic quantities are not in general described by tensors of rank two. A more elaborate analysis allow to estimate, in the framework of this model, the similarity between the mechanical and magnetic fabrics, and the validity of the approximation which consists of assimilating the magnetic quantities to such tensors.

Première partie : élaboration du modèle.

L'étude des propriétés magnétiques des roches déformées est un outil d'analyse structurale très performant et de plus en plus utilisé. Ce dernier ne remplace pas à lui seul les méthodes traditionnelles ; mais il est beaucoup moins lourd à mettre en œuvre et peut être utilisé en l'absence des marqueurs structuraux traditionnels.

Le but essentiel de ce travail est de montrer comment il est possible, à l'aide d'arguments simples : de justifier les relations observées entre l'anisotropie des propriétés magnétiques d'une roche et sa déformation interne et de les chiffrer dans le cas de l'orientation préférentielle de forme (O.P.F.).

\section{Aspect mécanique du modèle.}

Pour modéliser une roche polyminérale, on considère l'ensemble des grains d'un même minéral : les marqueurs et le reste de la roche : la matrice. On applique à cet ensemble le modèle hydrodynamique en se bornant au cas d'une déformation coaxiale orthorhombique isovolumique et en négligeant les interactions entre grains. La déformation finie s'exprime simplement par une transformation linéaire dans un système d'axes invariants au cours de celle-ci : les axes principaux de déformation. Le mouvement des marqueurs et l'invariance de leur nombre conduit à l'existence d'une densité d'orientation de leurs axes, définie dans le même repère sur la sphère de rayon unité. Cette grandeur contient toute l'information relative à l'O.P.F.

Les travaux de base relatifs à l'O.P.F. sont fort anciens (Jefferey, 1922 ; March, 1932) ; ils ont été cependant repris plus récemment (Gay, 1966, 1968 ;
Tullis, 1971, 1976 ; Owens, 1972 ; Reed et Tryggvason, 1974 ; Debat, 1975 ; Willis, 1977 ; Blanchard, 1979 ; Fernandez, 1984). Nous nous refèrerons essentiellement au travail de Fernandez [1] et nous nous bornerons à rappeler un certain nombre de résultats dus à cet auteur, ou qui en découlent simplement.

1.1 L'ORIENTATION PRÉFÉRENTIELLE DE FORME INDUITE PAR LA DÉFORMATION INTERNE DE LA ROCHE.

\subsubsection{Les hypothèses physiques du modèle développé par Fernandez.}

- On envisage un ensemble de marqueurs durs ayant la forme d'un ellipsoïde de révolution, dispersés dans une matrice homogène déformable ;

- la distribution initiale des marqueurs dans l'espace est uniforme ;

- leur concentration volumique moyenne est suffisamment faible pour que leurs interactions soient négligeables ;

- on súppose enfin que la déformation du système est homogène.

1.1.2 Mouvement de rotation d'un marqueur axial : cas de la déformation orthorhombique générale se produisant sans changement de volume. - Un marqueur axial est un grain ayant la forme d'un ellipsoïde de révolution allongé de demi-axes $a, b, c$ $(a=b<c)$; il est caractérisé par un paramètre de forme $\chi=\left(\frac{c}{a}\right)^{2}-1 /\left(\frac{c}{a}\right)^{2}+1$ et sa viscosité est grande par rapport à celle de la matrice (marqueur dur). La direction de l'axe de révolution est repérée en coordonnées sphériques par les angles $\theta, \phi$. 
Considérons un tel grain dont l'axe est défini par $\theta, \phi$ dans l'état initial non déformé. Après l'action d'un champ de déformation orthorhombique, l'axe du grain se retrouve dans l'état final déformé suivant $\theta^{\prime}, \phi^{\prime}$.

Les équations intégrées du mouvement qui définissent les relations entre $\theta, \phi$ et $\theta^{\prime}, \phi^{\prime}$ ont pour expression :

$$
\begin{gathered}
\frac{\operatorname{tg} \theta^{\prime}}{\operatorname{tg} \theta}\left(\frac{\sin 2 \phi^{\prime}}{\sin 2 \phi}\right)^{1 / 2}=\left(\lambda_{1} \lambda_{2}\right)^{3 x / 4} \\
\frac{\operatorname{tg} \phi^{\prime}}{\operatorname{tg} \phi}=\left(\frac{\lambda_{1}}{\lambda_{2}}\right)^{x / 2}
\end{gathered}
$$

Les élongations quadratiques principales $\lambda_{i}$ sont liées par la relation $\left(\lambda_{1} \lambda_{2} \lambda_{3}\right)^{1 / 2}=1$.

En faisant dans (1) $\lambda_{1} \neq \lambda_{2} ; \lambda_{3}=1$ ou $\lambda_{1}=$ $\lambda_{2} \neq \lambda_{3}$, on obtient les équations relatives au cas de la déformation plane coaxiale (selon Gay [2]) ou de la déformation coaxiale de révolution (selon Tullis [3] et Debat [4]).

1.1.3 Expression analytique de la densité $D$ dans le cas d'une déformation coaxiale orthorhombique. Le tableau I donne l'expression de $D$ dans les divers cas envisagés et précise le paramètre du modèle.
$D$ a une interprétation géométrique simple : c'est le cube du rayon vecteur d'un ellipsoïde obtenu par une opération de symétrie orthorhombique à partir de la sphère de rayon unité. Cet ellipsoïde n'est autre que l'ellipsoïde de fabrique introduit par Willis et tel que le définit Fernandez en généralisant le modèle de March. Pour cet auteur, la densité d'orientation d'un ensemble de marqueurs, tels que nous les avons définis, est identique à celle que donneraient des marqueurs de March $(\chi=1)$ pour une déformation fictive caractérisée par les élongations quadratiques principales $\Lambda_{i}$. Ces dernières sont fonctions des $\lambda_{i}$ caractérisant la déformation et de la forme des marqueurs. L'ellipsoïde de fabrique est alors identique à l'ellipsoïde de déformation finie de demi-axes $\left(\Lambda_{i}\right)^{1 / 2}\left({ }^{1}\right)$.

\section{Le choix des processus d'aimantation.}

Une roche polyminérale a en général, un comportement magnétique très complexe que l'on saurait

(1) Remarque : Nous avons retrouvé ce résultat par une autre méthode de calcul de la densité qui s'applique à une transformation conservant le système d'axes de référence et l'ensemble des directions transformées.

Tableau I. - Expression de la densité $D$ pour une déformation coaxiale orthorhombique isovolumique en coordonnées cartésiennes $\left(X^{\prime}, Y^{\prime}, Z^{\prime}\right)$ ou sphériques $\left(\theta^{\prime}, \phi^{\prime}\right)$. Le référentiel $\left(\mathrm{R}^{\prime}\right)$ est lié à l'état déformé. [Expressions of the $D$ for a coaxial, orthorhombic, isovolumic deformation in cartesian coordinates $\left(X^{\prime}, Y^{\prime}, Z^{\prime}\right)$ or spherical coordinates $\left(\theta^{\prime}, \phi^{\prime}\right)$. The reference frame $\left(\mathrm{R}^{\prime}\right)$ is linked to the deformed state.]

1) Quelconque : $\lambda_{1} \neq \lambda_{2} \neq 3$

$$
\begin{aligned}
D & =\left\{\lambda_{1}^{-x} X^{\prime 2}+\lambda_{2}^{-\chi} Y^{\prime 2}+\lambda_{3}^{-\chi} Z^{\prime 2}\right\}^{-3 / 2} \\
& =\left\{\lambda_{1}^{-\chi} \sin ^{2} \theta^{\prime} \cos ^{2} \phi^{\prime}+\lambda_{2}^{-\chi} \sin ^{2} \theta^{\prime} \sin ^{2} \phi^{\prime}+\lambda_{3}^{-\chi} \cos ^{2} \theta^{\prime}\right\}^{-3 / 2} .
\end{aligned}
$$

Le paramètre du modèle est une fonction de 2 des $3 \lambda_{i}$, ces derniers étant liés par la relation $\left(\begin{array}{lll}\lambda_{1} & \lambda_{2} & \lambda_{3}\end{array}\right)^{1 / 2}=1$.

2) Plane : $\lambda_{1} \neq \lambda_{2} ; \lambda_{3}=1$

$$
\begin{aligned}
D & =\left\{\lambda_{1}^{-x} X^{\prime 2}+\lambda_{2}^{-x} Y^{\prime 2}+Z^{\prime 2}\right\}^{-3 / 2} \\
& =\left\{k X^{\prime 2}+\frac{1}{k} Y^{\prime 2}+Z^{\prime 2}\right\}^{-3 / 2} \\
& =\left\{k \sin ^{2} \theta^{\prime} \cos ^{2} \phi^{\prime}+\frac{1}{k} \sin ^{2} \theta^{\prime} \sin ^{2} \phi^{\prime}+\cos ^{2} \theta^{\prime}\right\}^{-3 / 2} .
\end{aligned}
$$

Le paramètre du modèle est $k=\left(\frac{\lambda_{2}}{\lambda_{1}}\right)^{x / 2}$.

3) De révolution: $\lambda_{1}=\lambda_{2} \neq \lambda_{3}$

$$
\begin{aligned}
D & =\left\{\lambda_{3}^{\chi / 2}\left(X^{\prime 2}+Y^{\prime 2}\right)+\frac{1}{\lambda_{3}^{\chi}} Z^{\prime 2}\right\}^{-3 / 2} \\
& =\left\{\sqrt{k^{\prime}}\left(X^{\prime 2}+Y^{\prime 2}\right)+\frac{1}{k^{\prime}} Z^{\prime 2}\right\}^{-3 / 2} \\
& =\left\{\sqrt{k^{\prime}} \sin ^{2} \theta^{\prime}+\frac{1}{k^{\prime}} \cos ^{2} \theta^{\prime}\right\}^{-3 / 2}
\end{aligned}
$$

$D$ est indépendant de $\phi$, le problème étant de révolution autour de $\mathrm{OZ}$.

Le paramètre du modèle est : $k^{\prime}=\lambda_{3}^{\chi}$. 
éventuellement décrire à condition de connaître quantitativement la morphologie et les propriétés magnétiques de l'ensemble de ses constituants. Ce faisant on arriverait à une prévision rigoureuse de l'information magnétique globale ; mais le résultat aurait toutes les chances d'être inutilisable pour une comparaison avec l'expérience. Le cas concret que l'on doit rechercher, voire modéliser, est celui où la propriété qui intéresse le géologue ou le géophysicien peut être caractérisée à partir d'un très petit nombre de constituants, à condition toutefois que l'on sache acquérir l'information magnétique correspondante. De ce point de vue la minéralogie magnétique est essentielle.

Le modèle privilégie les processus d'aimantation. L'analyse qui suit a pour but de préciser certaines situations modélisables, de décrire les mécanismes d'aimantations adaptés et les grandeurs magnétiques pertinentes qui les caractérisent. Dans une étape ultérieure on peut affiner le modèle par une personnalisation des processus d'aimantation spécifiques des matériaux présents dans la roche.

2.1 NATURE DES CONSTITUANTS D'UNE ROCHE ET PROPRIÉTÉS MAGNÉTIQUES. - Les grains des divers minéraux constituant la roche peuvent être monocristallins ou polycristallins. Les premiers ont des axes de forme et des axes cristallographiques qui sont ou non liés, ou confondus. Quant aux seconds, les axes des cristallites qui les constituent peuvent avoir une orientation préférentielle par rapport aux axes de forme du grain ou au contraire être orientés au hasard. Les propriétés magnétiques des constituants sont linéaires ou non. Dans la première catégorie, on trouve les substances dia- et paramagnétiques, ces dernières pouvant être des composés au dessus de leur température d'ordre ou des grains fins superparamagnétiques $\left({ }^{2}\right)$. La susceptibilité suffit à elle seule à les caractériser et on peut la décrire par un tenseur symétrique de rang deux. Au contraire, les substances ordonnées ont un comportement non linéaire qui peut être doué de mémoire. Nous envisagerons essentiellement les ferrimagnétiques. Leur structure magnétique sera celle de monoou de polydomaines. Nous nous intéresserons à l'anisotropie de deux propriétés : la susceptibilité en champ faible et l'aimantation rémanente.

\subsection{L'ANISOTROPIE DE LA SUSCEPTIBILITÉ EN CHAMP FAIBLE.}

2.2.1 Les grains monocristallins. - Il faut d'emblée faire la distinction entre axes cristallographiques et axes de forme des grains. Dans le modèle de roche

(2) Remarque : On n'envisage ici que le comportement des grains fins superparamagnétiques en champs faibles. envisagé, l'O.P.F. résultant d'une certaine déformation est liée aux axes de forme des marqueurs. Par contre les propriétés magnétiques de ceux-ci sont liées aux axes cristallographiques et aux axes de forme.

Grains monocristallins monodomaines : Nous supposerons que les variations d'aimantation se produisent par rotation cohérente de l'aimantation et nous envisagerons deux situations. Dans la première, les axes de forme du grain coïncident avec les axes cristallographiques et l'axe de révolution du grain est un axe de facile aimantation. La susceptibilité initiale relative aux processus réversibles de rotation est proportionnelle à $\sin ^{2} \theta_{0}, \theta_{0}$ étant l'angle du champ magnétisant avec l'axe facile, que l'anisotropie magnétocristalline soit cubique ou uniaxiale. L'anisotropie de forme se comporte comme un terme uniaxial, petit devant l'énergie magnétocristalline. En ne considérant que ces deux termes énergétiques, l'anisotropie de la susceptibilité initiale du grain est représentée par un tenseur de rang 2 qui, rapporté à ses axes propres $X, Y, Z$, s'écrit :

$$
\left[\begin{array}{lll}
k & & \\
& k & \\
& & 0
\end{array}\right] \text {. }
$$

On a un axe de susceptibilité initiale nulle : l'axe de facile aimantation (ici $\mathrm{OZ}$ ) et un plan de susceptibilité initiale $(X O Y)$ qui lui est perpendiculaire. Dans ce plan, la susceptibilité est isotrope et sa valeur est maximale. Il est possible de calculer l'anisotropie de cette susceptibilité pour un ensemble de grains dont l'O.P.F. a une densité $D$.

Envisageons l'autre situation : le même ensemble de grains a des axes cristallographiques qui sont orientés au hasard par rapport aux axes de forme. La susceptibilité initiale est alors isotrope. On a $\overline{\sin }^{2} \theta_{0}=2 / 3$.

\section{Grains monocristallins polydomaines :}

a) Le grain idéalement polydomaine : Le grain est un monocristal sans défaut, ayant la forme d'un ellipsoïde de révolution et contenant un nombre suffisant de domaines pour qu'il soit possible de définir une aimantation moyenne. Sous l'influence d'un champ extérieur appliqué la structure en domaines réagit de manière à ce que le champ interne soit et reste nul. Ce dernier est la somme du champ appliqué et du champ créé par la matière aimantée elle-même. Ce champ démagnétisant de forme est calculable à condition de supposer l'aimantation uniforme et le grain limité par une surface du second degré. La nullité du champ interne fait que la susceptibilité dans la direction du champ appliqué est alors égale à l'inverse du coefficient de champ démagnétisant du grain dans cette même direction. 
Elle est liée aux axes de forme du grain et ne dépend pas des directions des axes cristallographiques par rapport à ces derniers pour une substance à anisotropie magnétocristalline cubique. Les premiers calculs de ce type ont été faits pour des substances massives par Néel [5] à propos de l'étude de l'aimantation du monocristal de fer $\left(K_{1}>0\right)$ et par Birss et Hegarty [6] dans le cas du monocristal de nickel $\left(K_{1}<0\right)$.

b) Les axes cristallographiques et les axes de forme du grain coïncident ; l'énergie magnétocristalline uniaxiale est prépondérante : on envisage essentiellement deux cas de figure, où l'énergie magnétocristalline domine toujours l'anisotropie de forme. Le premier correspond à $K_{\mathrm{u}}$ (constante d'anisotropie uniaxiale) positif. L'axe de facile aimantation coïncide avec l'axe du grain, le plan perpendiculaire à cet axe est un plan de difficile aimantation. Pour un champ dirigé suivant une direction quelconque de ce plan, on a un processus de rotation réversible en champ faible. Par contre, pour un champ dirigé suivant l'axe de facile aimantation, on a un mécanisme de déplacement de parois à $180^{\circ}$; si toutefois on néglige les domaines de fermeture. Un tel comportement conduit à une loi de Rayleigh. On peut alors représenter la susceptibilité initiale par un tenseur de rang 2. La susceptibilité en champs faibles peut l'être également, mais de façon approchée. Avec les notations précédentes et rapporté à ses axes propres $X, Y, Z$, le tenseur s'écrit :

$$
\left[\begin{array}{lll}
k_{1} & & \\
& k_{2} & \\
& & k_{3}
\end{array}\right]
$$

avec $k_{1}=k_{2}<k_{3}$, l'axe du grain étant suivant $\mathrm{O} Z$. $\mathrm{OZ}$ est l'axe de plus grande susceptibilité. $X O Y$ est le plan de plus petite susceptibilité, celle-ci est isotrope dans ce plan.

Une analyse analogue s'applique à $K_{\mathrm{u}}<0$. L'axe de difficile aimantation coïncide avec l'axe de révolution du grain et le plan équatorial est un plan de facile aimantation. Le tenseur traduisant l'anisotropie de la susceptibilité s'écrit avec les mêmes notations :

$$
\left[\begin{array}{lll}
k_{1} & & \\
& k_{1} & \\
& & k_{3}
\end{array}\right]
$$

avec $k_{1}>k_{3}$.

c) Les axes de forme ne coïncident pas avec les axes cristallographiques.

Ce cas est nettement plus complexe et il ne nous semble pas judicieux de l'aborder dans une première étude.
2.2.2 Les grains polycristallins. - Il s'agit ici uniquement de polydomaines ayant en général des propriétés isotropes, du moins si les axes cristallographiques des cristallites sont orientés au hasard par rapport aux axes de forme du grain. Il est raisonnable de penser que chaque grain va suivre une loi de Rayleigh et que l'effet de forme sera prépondérant dans la susceptibilité en champ faible. On peut alors calculer pour un ensemble de grains l'anisotropie de la susceptibilité résultant d'une O.P.F. Nous n'envisagerons pas ici le cas où il existe une orientation préférentielle des axes cristallographiques des cristallites par rapport aux axes de forme du grain.

\subsection{L'ANISOTROPIE DE L'AIMANTATION RÉMA-} NENTE. - Considérons un ensemble de grains à température constante $T$ et appliquons un champ magnétique suffisant pour les saturer. Les grains sont alors monodomaines, leur aimantation étant alignée avec le champ, ou du moins très voisine de celui-ci. Coupons le champ et examinons quelques possibilités. Il s'agit là d'un processus d'ARIS.

Pour un grain fin idéal, tel que nous l'envisagerons par la suite, l'aimantation relaxe suivant la direction de facile aimantation la plus proche du champ magnétisant et le grain reste aimanté à saturation dans cette direction à condition toutefois que $T<T_{\mathrm{B}}$. L'information magnétique s'exploite sans difficulté : cf. 2.2.2. $T_{\mathrm{B}}$ est la température de blocage du grain. Au-dessous de celle-ci, l'agitation thermique ne suffit plus à elle seule à retourner l'aimantation du grain. Par contre si $T>T_{\mathrm{B}}$, le grain devient superparamagnétique.

Pour un polydomaine idéal, l'aimantation rémanente est nulle : on perd toute l'information.

Pour un grain initialement polydomaine, il y a deux possibilités après saturation et retour en champ nul : il reste monodomaine ou non. Dans le premier cas nous avons affaire à un pseudo-monodomaine. Ces derniers ont fait l'objet de nombreuses études de la part des spécialistes du paléomagnétisme car ils sont porteurs d'information au même titre que les grains fins monodomaines. La nucléation d'une paroi nécessite l'application d'un champ inverse. Elle résulte d'un équilibre énergétique local où le champ démagnétisant de forme joue un rôle prépondérant. Dans ce qui suit nous n'envisagerons pas le cas de ces objets.

Pour les autres grains la nucléation en champ nul est essentiellement gouvernée par les défauts du cristal. C'est le cas dans les phénomènes d'hystérésis habituels. On peut cependant observer dans des cristaux sans défauts de l'hysteresis topologique [7]. L'aimantation rémanente observée est liée à la structure en domaines qui apparaît en champ nul, cette dernière étant fortement dépendante des défauts du grain au sens habituel ou topologique du terme. Un processus d'aimantation du type lois de 
Rayleigh prend en compte les défauts au sens habituel. L'anisotropie de l'aimantation rémanente dans le domaine de Rayleigh peut être une grandeur intéressante à considérer. On y a très simplement accès par la mesure de la susceptibilité irréversible en champ faible (cf. 3.4).

L'A.T.R. a été étudiée presque exclusivement pour les grains fins ou des pseudo-monodomaines. Elle donne dans ce cas des informations comparables et complémentaires de l'ARIS (cf. 3.2.3).

L'aimantation thermorémanente d'une structure en domaines dans un matériau massif a été rarement étudiée [8] ; quant à son anisotropie elle ne l'a pas été à notre connaissance.

2.4 LES PROCESSUS D'AIMANTATION RETENUS. Nous envisagerons trois cas limite de grains :

a) Le grain fin idéal : c'est un monocristal monodomaine, ayant la forme d'un ellipsoïde allongé, l'axe de révolution étant un axe de facile aimantation. Les axes cristallographiques et les axes de forme coïncident. Il est aimanté à saturation ; son moment peut tourner sous l'influence d'un champ appliqué par rotation cohérente des moments élémentaires. C'est le mécanisme le plus simple que l'on puisse envisager pour décrire les rotations réversibles ou irréversibles du moment.

b) Le polydomaine idéal : le processus d'aimantation du polydomaine idéal est celui décrit en 2.2.1. Il faut bien être conscient que ces deux cas correspondent à des hypothèses limite. Envisageons par exemple, un ensemble de grains de magnétite à granulométrie étendue. Pour des tailles croissantes de ceuxci, on observe des monodomaines, des grains comportant quelques domaines et enfin des vrais polydomaines. Dans les marqueurs comportant quelques domaines, ceux-ci sont fortement couplés entre eux par des effets magnétostatiques et leurs processus d'aimantation ne sont pas d'un accès facile. Par ailleurs ils n'ont pas en général la forme d'ellipsoïdes. Après saturation et retour en champ nul, ils peuvent avoir un comportement de pseudo-monodomaine.

c) Le grain suit une loi de Rayleigh : la nature magnétique du grain est telle que son aimantation soit décrite par une loi du type :

$$
M=a h+b h^{2}
$$

où $h$ est le champ interne et $a$ et $b$ la susceptibilité réversible et la constante de Rayleigh. Cette loi n'est en général pas valable dans les champs très faibles ou des phénomènes comme l'agitation thermique, deviennent prépondérants et ne sont pas pris en compte dans les modèles. Le champ $h$ doit par ailleurs rester faible devant le champ coercitif, ce qui signifie simplement que la structure en domaines ne doit pas subir de modifications profondes au cours de son évolution. Ce choix est moins académique qu'il n'y paraît. De nombreux objets très différents ont un tel comportement. Nous en citerons quelques-uns : une paroi de Bloch unique se déplaçant dans un milieu aléatoirement perturbé par des défauts, un polycristal, un ensemble de «grains de Preisach » fortement couplés entre eux par des interactions convenables... Les lois de Rayleigh sont des lois limites traduisant l'évolution d'une structure en domaines caractérisée par une faible métastabilité.

De même que nous avons négligé les interactions mécaniques entre grains dans le modèle hydrodynamique, nous négligerons également les interactions magnétiques entre marqueurs.

\section{Expressions de l'anisotropie de quelques proprié- tés magnétiques résultant de l'O.P.F.}

3.1 PRINCIPE DU CALCUL. - Tous les grains de l'ensemble sont identiques et sans interactions entre eux. On calcule la propriété pour un grain et on somme sur l'ensemble compte tenu de l'O.P.F.

Ce faisant on est conduit à évaluer des intégrales doubles scalaires ou vectorielles définies sur une demi-sphère de rayon unité ; en effet un grain est caractérisé par l'orientation de son axe de révolution : $\pm \mathbf{u}(\theta, \phi)$ définit le même grain. Le choix des limites d'intégration en $\theta$ et $\phi$ est caractéristique de la propriété envisagée, nous le noterons symboliquement $1 / 2$ Sp. Pour une direction déterminée $\theta, \phi$, à laquelle correspond l'élément $\mathrm{d} S$ sur la sphère de rayon unité, il y a $\mathrm{d} N$ grains : $\mathrm{d} N=D_{0} D(\theta, \phi) \mathrm{d} S$. Le nombre total de ceux-ci vaut:

$$
\begin{aligned}
N & =\iint_{1 / 2 \mathrm{Sp}} D_{0} D(\theta, \phi) \mathrm{d} S \\
& =\iint_{1 / 2 \mathrm{Sp}} D_{0} D(\theta, \phi) \sin \theta \mathrm{d} \theta \mathrm{d} \phi .
\end{aligned}
$$

Le champ magnétisant a une direction $\mathbf{h}\left(\theta_{0}, \phi_{0}\right)$ et la propriété considérée pour un grain dans la direction $\theta, \phi$ vaut : $p(\theta, \phi)$. La propriété relative à l'ensemble s'écrit :

$$
P_{\mathrm{h}}=\iint_{1 / 2 \mathrm{Sp}} p(\theta, \phi) D_{0} D(\theta, \phi) \mathrm{d} S .
$$

Pour pouvoir comparer l'ensemble des propriétés étudiées entre elles, il faut définir une propriété réduite caractéristique de la seule O.P.F. Nous avons choisi :

$$
\mathfrak{T}_{\mathrm{h}}=\left(\frac{P}{P_{0}}\right)_{\mathrm{h}}
$$

$P_{0}$ étant la propriété envisagée pour le même ensemble de grains dans le cas d'une répartition isotrope des directions de leurs axes. La densité $D_{0}$ vaut alors $N / 2 \pi$. 
On a finalement :

$\mathfrak{T}_{\mathbf{h}}=$

$$
=\frac{2 \pi \iint_{1 / 2 \mathrm{Sp}} P(\theta, \phi) D(\theta, \phi) \mathrm{d} S}{\left\{\iint_{1 / 2 \mathrm{sp}} P(\theta, \phi) \mathrm{d} S\right\}\left\{\iint_{1 / 2 \mathrm{Sp}} D(\theta, \phi) \mathrm{d} S\right\}}
$$

On fait ensuite varier la direction de h. Si la propriété est un scalaire on trace l'indicatrice de la grandeur considérée en fonction de la direction de $\mathbf{h}$. Si la propriété est un vecteur on détermine ses composantes et on repasse en coordonnées sphériques ce qui permet d'obtenir $|\mathfrak{T}| ; \theta_{1} ; \phi_{1}$; et le déphasage caractérisé par l'angle $(\mathbf{h}, \mathfrak{T})$. On trace également l'indicatrice de $\mathfrak{T}$.

Comme nous avons utilisé comme repère les axes principaux de déformation, il est possible de comparer simplement l'effet et la cause, c'est-à-dire l'indicatrice à l'ellipsoïde de déformation finie.

Nous donnons en annexe quelques exemples de ce type de calcul. Les intégrales ainsi définies n'ont, en général, pas de solution analytique évidente, aussi les avons-nous calculées numériquement.

Nous envisageons successivement un ensemble de grains fins, de polydomaines, ou de marqueurs suivant une loi de Rayleigh.

\subsection{PROPRIÉTÉS D'UN ENSEMBLE DE GRAINS FINS.}

3.2.1 Anisotropie de la susceptibilité initiale (cf. 2.2.1). - Dans le cas où l'anisotropie magnétocristalline des grains est uniaxiale, la susceptibilité initiale de rotation s'écrit :

$$
s_{\mathrm{i}}=\frac{M_{\mathrm{s}}^{2} \sin ^{2} \alpha}{2 K_{\mathrm{u}}} .
$$

$M_{\mathrm{s}}$ étant l'aimantation à saturation du matériau, $\alpha$ l'angle du champ magnétisant avec la direction de facile aimantation et $K_{\mathrm{u}}$ la constante d'anisotropie. Pour un grain à anisotropie cubique on a :

$$
\begin{aligned}
& K_{1}>0 \quad s_{\mathrm{i}}=\frac{M_{\mathrm{s}}^{2} \sin ^{2} \alpha}{2 K_{1}} \\
& K_{1}<0 \quad s_{\mathrm{i}}=\frac{-3 M_{\mathrm{s}}^{2} \sin ^{2} \alpha}{4 K_{1}} \quad \text { cf. [9]. }
\end{aligned}
$$

Remarquons que le terme énergétique dû à l'anisotropie de forme se comporte comme un terme uniaxial. Dans les trois cas envisagés on a $s_{\mathrm{i}}=$ $k \sin ^{2} \alpha$. Pour un ensemble de grains caractérisé par une orientation préférentielle des axes de forme, de densité $D(\theta, \phi)$, on obtient :

$$
\begin{aligned}
\left(s_{\mathrm{i}}\right)_{\mathbf{h}} & =\frac{\left(S_{\mathrm{i}}\right)_{\mathrm{h}}}{\left(S_{\mathrm{i}}\right)_{0}} \\
& =\frac{3 \int_{0}^{\pi / 2} \int_{0}^{2 \pi}\left[1-(\mathbf{u} \cdot \mathbf{h})^{2}\right] D(\theta, \phi) \mathrm{d} S}{2 \int_{0}^{\pi / 2} \int_{0}^{2 \pi} D(\theta, \phi) \mathrm{d} S}
\end{aligned}
$$

avec: $\mathbf{u}(\theta, \phi)$ vecteur unitaire courant et $\mathbf{h}\left(\theta_{0}, \phi_{0}\right)$ vecteur unitaire du champ dans la direction duquel on détermine la susceptibilité relative. Remarquons que dans le cas où les axes cristallographiques du grain ont une répartition isotrope par rapport à ses axes de forme, on a un milieu isotrope de susceptibilité initiale $2 / 3 k N, N$ étant le nombre de grains.

\subsubsection{Anisotropie de l'aimantation rémanente} isotherme à saturation ARIS. - A une température $T$ on applique un champ magnétique $H$ dirigé suivant $\mathbf{h}\left(\theta_{0}, \phi_{0}\right)$ d'amplitude suffisante pour aligner l'ensemble des moments des grains. On coupe le champ et on étudie l'aimantation rémanente résultant de ce traitement. Il y a une seule espèce de grains, c'est-à-dire une seule température de blocage $T_{\mathrm{B}}$; on suppose $T<T_{\mathrm{B}}$. Après coupure du champ les moments des grains se placent suivant la direction de facile aimantation la plus proche du champ.

L'aimantation rémanente $\mathbf{M}_{\mathrm{r}}$, moment magnétique de l'unité de volume s'écrit :

$$
\mathbf{M}_{\mathrm{r}}=\mu \iint_{1 / 2 \mathrm{Sph}} \mathbf{u}(\theta, \phi) D_{0} D(\theta, \phi) \mathrm{d} S,
$$

où $\mu$ est le moment d'un grain, dans les conditions de l'expérience, ramené à l'unité de volume. $1 / 2 \mathrm{Sp} \mathrm{h}$ définit les limites d'intégration sur $\theta$ et $\phi$ correspondant à une demi-sphère de plan équatorial perpendiculaire à $\mathbf{h}$ et de pôle suivant $\mathbf{h}$.

On a pour l'aimantation relative $\left(m_{\mathrm{r}}\right)_{\mathrm{h}}$ :

$$
\begin{aligned}
\left(\mathbf{m}_{\mathrm{r}}\right)_{\mathbf{h}} & =\frac{\left(\mathbf{M}_{\mathrm{r}}\right)_{\mathbf{h}}}{\left(M_{\mathrm{r}}\right)_{0}} \\
& =2 \frac{\iint_{1 / 2 \mathrm{Sph}} \mathbf{u}(\theta, \phi) D(\theta, \phi) \mathrm{d} S}{\iint_{1 / 2 \mathrm{Sph}} D(\theta, \phi) \mathrm{d} S}
\end{aligned}
$$$$
\text { avec : }\left(M_{\mathrm{r}}\right)_{0}=\frac{N \mu}{2} \text {. }
$$

\subsubsection{Anisotropie de l'aimantation thermorémanente} en champ faible: ATR. - L'échantillon est refroidi sous champ magnétique depuis une température supérieure à la température de Curie, jusqu'à la température $T$ de l'expérience. $T<T_{\mathrm{B}}$. 
On a :

$$
\begin{aligned}
&\left(M_{\mathrm{TR}}\right)_{\mathbf{h}}=\mu \iint_{\substack{1 / 2 \mathrm{Sph}\\
}} \mathbf{u}(\theta, \phi) \text { th }\left\{a_{\mathrm{L}}(\mathbf{u} \cdot \mathbf{h})\right\} \times \\
& \times D_{0} D(\theta, \phi) \mathrm{d} S \quad \text { (cf. Néel [10]) } .
\end{aligned}
$$

$a_{\mathrm{L}}$ est la variable de Langevin $\frac{\mu H}{k T}$. Pour $a_{\mathrm{L}}$ petit devant 1 on développe th $\left\{a_{\mathrm{L}}(\mathbf{u} \cdot \mathbf{h})\right\}$ et on se borne au premier terme du développement. On obtient :

$$
\left(M_{\mathrm{TR}}\right)_{\mathbf{h}}=\mu a_{\mathrm{L}} \iint_{1 / 2 \mathrm{Sph}} \mathbf{u}(\mathbf{u} \cdot \mathbf{h}) D_{0} D(\theta, \phi) \mathrm{d} S .
$$

On a finalement pour l'aimantation thermorémanente en champ faible, rapporté à $\left(M_{\mathrm{TR}}\right)_{0}$ :

$$
\begin{aligned}
\left(\mathbf{m}_{\mathrm{TR}}\right)_{\mathbf{h}} & =\frac{\left(\mathbf{M}_{\mathrm{TR}}\right)_{\mathbf{h}}}{\left(M_{\mathrm{TR}}\right)_{0}} \\
& =3 \frac{\iint_{1 / 2 \mathrm{Sph}} \mathbf{u}(\mathbf{u} \cdot \mathbf{h}) D(\theta, \phi) \mathrm{d} S}{\iint_{1 / 2 \mathrm{Sph}} D(\theta, \phi) \mathrm{d} S}
\end{aligned}
$$

avec : $\left(M_{\mathrm{TR}}\right)_{0}=N \mu \frac{a_{\mathrm{L}}}{3}$.

3.2.4 Conséquence du caractère non linéaire et de la mémoire du milieu constitué par l'ensemble de grains fins précédemment défini : anisotropie magnétique résultant de la déformation du milieu aimanté: $A R I S ̉ D, A T R D$. - L'ensemble de grains fins tel que nous l'avons défini est un milieu non linéaire doué de mémoire. L'anisotropie magnétique enregistrée par celui-ci, pour une déformation finie donnée, dépend de l'ordre dans lequel ont été effectuées les opérations d'aimantation et de déformation. Il n'est pas indifférent d'aimanter la substance et de la déformer ensuite, ou d'aimanter celle-ci après l'avoir déformée.

Pour illustrer ce propos calculons l'anisotropie obtenue en déformant le milieu aimanté. Dans une telle opération la direction du champ magnétique $\mathbf{h}\left(\theta_{0}, \phi_{0}\right)$ devient $\mathbf{h}_{D}$ et le vecteur courant $\mathbf{u}$ devient $\mathbf{u}_{D}$. Les équations intégrées du mouvement donnent l'évolution des angles $\theta$ et $\phi$ au cours de la déformation (cf. 1.2, relation (1)).

En ce qui concerne l'ARIS, il suffit de changer les limites d'intégration. L'indicatrice de l'ARISD est identique à celle de l'ARIS. Par contre le déphasage, qui est l'angle entre $\mathbf{h}\left(\theta_{0}, \phi_{0}\right)$ et $\mathbf{M}_{\mathrm{r}}$ change.

Dans le calcul de l'ATR, il faut à la fois changer les limites d'intégration et remplacer $(\mathbf{u} \cdot \mathbf{h})$ par $\left(\mathbf{u}_{D} \cdot \mathbf{h}_{D}\right)$.

Dans ce dernier cas il y a changement de l'indicatrice et du déphasage.

Ce résultat a une implication directe en paléomagnétisme [11] et montre par ailleurs que l'aimanta- tion naturelle d'une roche dépend à la fois de son histoire magnétique et tectonique.

\subsection{PROPRIÉTÉS D'UN ENSEMBLE DE GRAINS IDÉA-} LEMENT POLYDOMAINES. ANISOTROPIE DE LA SUSCEPTIBILITÉ EN CHAMP FAIBLE. - On envisage un ensemble de marqueurs polydomaines identiques et sans interaction entre eux. L'anisotropie due à l'O.P.F. des marqueurs résulte uniquement d'un effet de forme. Le calcul donné dans l'annexe 1 conduit à l'expression de la susceptibilité relative dans la direction $\mathbf{h}$ du champ appliqué.

On obtient :

$$
\begin{aligned}
S_{\mathrm{h}}=\frac{\left(S_{\mathrm{c}}\right)_{\mathrm{h}}}{\left(S_{\mathrm{c}_{0}}\right)_{\mathrm{h}}}= & \\
= & \frac{2 \pi \int_{0}^{\pi / 2} \int_{0}^{2 \pi} F(\theta, \phi) D(\theta, \phi) \mathrm{d} S}{\left\{\int_{0}^{\pi / 2} \int_{0}^{2 \pi} F(\theta, \phi) \mathrm{d} S\right\} \times} \\
& \times\left\{\int_{0}^{\pi / 2} \int_{0}^{2 \pi} D(\theta, \phi) \mathrm{d} S\right\}
\end{aligned}
$$

où :

$$
F(\theta, \phi)=\left\{\left(1-N_{3}\right)+\left(3 N_{3}-1\right)(\mathbf{u} \cdot \mathbf{h})^{2}\right\}^{-1}
$$

$\left(S_{\mathrm{c}}\right)_{\mathbf{h}}$ est la susceptibilité de l'ensemble des grains orientés dans la direction du champ appliqué; $\left(S_{\mathrm{e}_{0}}\right)_{\mathrm{h}}$ la même grandeur pour l'ensemble orienté au hasard, $N_{3}$ le coefficient de champ démagnétisant du grain suivant l'axe de révolution, u le vecteur courant et $\mathbf{h}$ le vecteur unitaire du champ appliqué.

\subsection{PROPRIÉTÉ D'UN ENSEMBLE DE GRAINS PRÉSEN-} TANT UNE O.P.F. DANS LE CAS OÙ L'AIMANTATION DE CHACUN D'EUX EST RÉGIE PAR UNE LOI DE RAYLEIGH. - Le matériau constituant le marqueur est magnétiquement isotrope. L'anisotropie résulte de l'existence du champ démagnétisant. C'est un effet de forme; il est calculable du fait que la loi d'aimantation est connue et que les grains sont des ellipsoïdes.

Le calcul est donné dans l'annexe 2.

La susceptibilité réversible relative dans la direction du champ appliqué $\mathbf{h}_{0}=\mathbf{u}_{0} h_{0}$ a pour expression :

$$
\left(S_{\mathrm{r}}\right)_{\mathrm{h}_{0}}=\frac{3}{2 a_{1}^{\prime}+a_{3}^{\prime}} \frac{\int_{0}^{\pi / 2} \int_{0}^{2 \pi} F(\theta, \phi) D(\theta, \phi) \mathrm{d} S}{\int_{0}^{\pi / 2} \int_{0}^{2 \pi} D(\theta, \phi) \mathrm{d} S}
$$

où $F(\theta, \phi)=\left\{a_{1}^{\prime}+\left(a_{3}^{\prime}-a_{1}^{\prime}\right)\left(\mathbf{u} \cdot \mathbf{u}_{0}\right)^{2}\right\}$,

$\mathbf{u}$ est le vecteur unitaire courant, $\mathbf{u}_{0}$ le vecteur du champ $\mathbf{h}_{0}$ et où les $a_{i}^{\prime}$ sont les susceptibilités réversi- 
bles effectives suivant les axes du grain. Ce sont des fonctions de $a$ et des $N_{i}$ :

$$
a_{i}^{\prime}=\frac{a}{1+a N_{i}}
$$

La susceptibilité irréversible relative dans la direction du champ appliqué $\mathbf{h}_{0}$ s'écrit :

$$
\left(S_{\mathrm{ir}}\right)_{\mathrm{h}_{0}}=\frac{1}{L} \frac{\int_{0}^{\pi / 2} \int_{0}^{2 \pi}\left(S_{1 \text { ir }}\right)_{\mathrm{h}_{0}} D(\theta, \phi) \mathrm{d} S}{\int_{0}^{\pi / 2} \int_{0}^{2 \pi} D(\theta, \phi) \mathrm{d} S}
$$

où : $L=\left\{\frac{b_{3}^{\prime}}{8} Z_{0}\left(3-Z_{0}^{2}\right)-b_{2}^{\prime} \frac{3 \pi}{32} Z_{0}\left(1+Z_{0}^{2}\right)\right\}$, $\left(S_{1 \text { ir }}\right)_{\mathrm{h}_{0}}$ est la susceptibilité d'un grain et les $b_{i}^{\prime}$ les constantes de Rayleigh effectives suivant les axes de celui-ci. On a :

$$
b_{i}^{\prime}=\frac{b}{1+a N_{i}}\left(1-\frac{2 a N_{i}}{\left(1+a_{i} N_{i}\right)^{2}}+\frac{a^{2} N_{i}}{\left(1+a N_{i}\right)^{3}}\right) .
$$

L'aimantation rémanente relative est identique: à la susceptibilité relative irréversible. En effet :

$$
M_{\mathrm{r}}=\frac{b}{2} h_{M}^{2} \text {, soit }\left(m_{\mathrm{r}}\right)_{\mathrm{h}_{0}}=\frac{\left(M_{\mathrm{r}}\right)_{\mathrm{h}_{0}}}{\left(M_{\mathrm{r}}\right)_{0}}=\left(S_{\mathrm{ir}}\right)_{\mathrm{h}_{0}} \text {. }
$$

On détermine également sans difficulté le vecteur aimantation relative à partir de ses composantes. Le passage en coordonnées sphériques permet d'avoir son module et les angles $\phi$ et $\boldsymbol{\theta}$ correspondants.

Remarquons qu'il est possible de déterminer expérimentalement le terme réversible et le terme irréversible de la susceptibilité. En ce qui concerne le terme réversible on a l'équivalent d'un milieu linéaire.

\section{Anisotropie des propriétés magnétiques et aniso- tropie de la déformée.}

Le modèle donne accès aux indicatrices de la susceptibilité relative dans la direction du champ magnétisant et de l'aimantation rémanente relative en fonction de la direction du champ magnétique. Les axes de référence sont les axes principaux de déformation.

Par ailleurs le modèle de déformation finie utilisée est caractérisé par une transformation linéaire qui exprimée dans ce système d'axes s'écrit :

$$
\left[\begin{array}{l}
X_{0}^{2} \\
Y_{0}^{2} \\
Z_{0}^{2}
\end{array}\right]=\left[\begin{array}{ccc}
\frac{1}{\lambda_{1}} & & \\
& \frac{1}{\lambda_{2}} & \\
& & \frac{1}{\lambda_{3}}
\end{array}\right]\left[\begin{array}{l}
\rho^{2} X^{\prime 2} \\
\rho^{2} Y^{\prime 2} \\
\rho^{2} Z^{\prime 2}
\end{array}\right] .
$$

Elle fait passer de la sphère de rayon unité dans le milieu non déformé : $X_{0}^{2}+Y_{0}^{2}+Z_{0}^{2}=1$ à l'ellipsoïde de déformation finie : $\frac{1}{\rho^{2}}=\frac{X^{\prime 2}}{\lambda_{1}}+\frac{Y^{\prime 2}}{\lambda_{2}}+\frac{Z^{\prime 2}}{\lambda_{3}}$ et fait correspondre à toute direction $\left(\theta_{0}, \phi_{0}\right)$ avant déformation une direction $\left(\theta^{\prime}, \phi^{\prime}\right)$ de la déformée. $\theta^{\prime}$ et $\phi^{\prime}$ s'expriment en fonction de $\theta_{0}$ et $\phi_{0}$ à partir des expressions des $X_{0}^{2}, Y_{0}^{2}, Z_{0}^{2}$. Ces équations doivent être vérifiées quelque soit le couple $\left(\theta_{0}\right.$, $\left.\phi_{0}\right)$.

Pour faciliter la comparaison entre l'anisotropie des propriétés magnétiques résultant du modèle et l'anisotropie de la déformation finie, on est amené à se demander dans quelle mesure il est possible d'approximer la susceptibilité par un tenseur de rang deux et le modèle d'aimantation rémanente par une transformation linéaire analogue à la précédente.

4.1 CAS DE LA SuSCEPTIBILITÉ. - Si une propriété $S$ est décrite par un tenseur de rang deux : $P_{i}=$ $\left[S_{i j}\right] q_{j}$, l'intensité de celle-ci dans une certaine direction s'obtient en appliquant $q$ dans cette direction et en mesurant $p_{\|} / q, p_{\|}$étant la composante de p suivant q. L'expression analytique de $S$ par rapport aux axes principaux de $\left[S_{i j}\right]$ et pour une direction de q caractérisée par $l_{1}, l_{2}, l_{3}$ s'écrit :

$$
S=l_{1}^{2} S_{1}+l_{2}^{2} S_{2}+l_{3}^{2} S_{3}
$$

où l'on a : $S_{1}=S_{11} ; S_{2}=S_{22} ; S_{3}=S_{33}$.

Pour des axes quelconques on a : $S=S_{i j} l_{i} l_{j}$.

Nous allons comparer l'indicatrice de la susceptibilité $K$ donnée par le modèle à l'équation $S=$ $S_{i i} l_{i}^{2}$.

Pour ce faire développons l'indicatrice de $K$ en série de Fourier [12]. On a :

$$
\begin{aligned}
& K=\sum_{\substack{i=0 \\
j=0}}^{\infty} a_{i j} \cos i \theta_{0} \cos j \phi_{0}+b_{i j} \sin i \theta_{0} \cos j \phi_{0}+ \\
& \quad+c_{i j} \cos i \theta_{0} \sin j \phi_{0}+d_{i j} \sin i \theta_{0} \sin j \phi_{0}
\end{aligned}
$$

$\boldsymbol{\theta}_{0}$ et $\phi_{0}$ caractérisant la direction du champ. Les symétries orthorhombiques de la densité font que seuls les harmoniques pairs du premier terme subsistent.

$$
K=\sum_{\substack{i=0 \\ j=0 \\ i \text { et } j \text { pairs }}}^{\infty} a_{i j} \cos i \theta_{0} \cos j \phi_{0}
$$

Le développement de l'indicatrice $S$ correspondant à une propriété tensorielle s'écrit :

$$
\begin{aligned}
S=a_{00}+a_{20} \cos 2 \theta_{0}+a_{02} & \cos 2 \phi_{0}+ \\
& +a_{22} \cos 2 \theta_{0} \cos 2 \phi_{0} .
\end{aligned}
$$

Les termes du développement de $K$ autres que ceux figurant dans celui de $S$ représentent l'écart au milieu linéaire caractérisé par un tenseur de rang deux. 
Si l'importance de ces termes est négligeable vis-àvis de celle des termes en $a_{00}, a_{20}, a_{22}$ on peut faire l'approximation du milieu linéaire.

Les susceptibilités principales ont alors pour expression :

$$
\begin{aligned}
& S_{1}=\left(a_{00}-a_{20}\right)+2 a_{22} \\
& S_{2}=\left(a_{00}-a_{20}\right)-2 a_{22} \\
& S_{3}=\left(a_{00}+a_{20}\right) .
\end{aligned}
$$

Il est alors possible de comparer l'ellipsoïde des susceptibilités à l'ellipsoïde de déformation finie.

La simple étude de la différence :

$$
\begin{aligned}
K-\left\{K_{1} \sin ^{2} \theta_{0} \cos ^{2} \phi_{0}+K_{2} \sin ^{2} \theta_{0} \sin ^{2} \phi_{0}+\right. \\
\left.+K_{3} \cos ^{2} \theta_{0}\right\}
\end{aligned}
$$

en fonction de $\theta_{0}, \phi_{0}$, renseigne immédiatement sur la validité de l'hypothèse du milieu linéaire. On prend pour $K_{1}, K_{2}, K_{3}$ les valeurs de $K$ suivant les axes $X, Y, Z$ quand le champ est également dirigé suivant ces axes.

4.2 CAS DE L'Aimantation RÉmanente. - Ce modèle donne pour chaque couple $\theta_{0}, \phi_{0}$ caractérisant la direction du champ magnétisant le vecteur $\mathbf{m}_{\mathrm{r}}$ caractérisé par son module $m_{\mathrm{r}}$ et les angles $\boldsymbol{\theta}_{1}^{\prime}, \boldsymbol{\phi}_{1}^{\prime}$.

Considérons la quantité $\frac{1}{m_{\mathrm{r}}^{2}}$ et développons-la en série de Fourier. On a une expression analogue à celle du cas précédent soit :

$$
\frac{1}{m_{\mathrm{r}}^{2}}=\sum_{\substack{i=0 \\ j=0 \\ i \text { ct } j \text { pairs }}}^{\infty} a_{i j} \cos i \theta_{1}^{\prime} \cos j \phi_{1}^{\prime}
$$

Le développement en série de Fourier d'un ellipsoïde de rayon $m_{\mathrm{r}}^{\prime}$ s'écrit :

$$
\begin{aligned}
\frac{1}{m_{\mathrm{r}}^{\prime 2}}=a_{00}+a_{20} \cos 2 \theta^{\prime}+ & a_{02} \\
& \cos 2 \phi^{\prime}+ \\
& +a_{22} \cos 2 \theta^{\prime} \cos 2 \phi^{\prime} .
\end{aligned}
$$

Les termes du développement de $\frac{1}{m_{\mathrm{r}}^{2}}$ autres que ceux figurant dans le développement de $\frac{1}{m_{\mathrm{r}}^{\prime 2}}$ représentent l'écart de l'indicatrice à l'ellipsoïde. Si ce dernier est négligeable la première condition pour que le modèle se ramène à une transformation linéaire analogue à celle définissant la déformation finie est satisfaite. Mais il faut également que l'indicatrice soit décrite comme elle le serait dans une transformation linéaire. Pour cela on doit avoir :

$$
\sin ^{2} \theta_{0} \cos ^{2} \phi_{0}=\frac{m_{\mathrm{r}}^{\prime 2}}{M_{1}^{\prime 2}} \sin ^{2} \theta_{1}^{\prime} \cos ^{2} \phi_{1}^{\prime}
$$

$$
\begin{aligned}
\sin ^{2} \theta_{0} \sin ^{2} \phi_{0} & =\frac{m_{\mathrm{r}}^{\prime 2}}{M_{2}^{\prime 2}} \sin ^{2} \theta_{1}^{\prime} \sin ^{2} \phi_{1}^{\prime} \\
\cos ^{2} \theta_{0} & =\frac{m_{\mathrm{r}}^{\prime 2}}{M_{3}^{\prime 2}} \cos ^{2} \theta_{1}^{\prime} .
\end{aligned}
$$

Ce système d'équations définissant le couple $\left(\theta^{\prime}, \phi^{\prime}\right)$ à partir de $\theta_{0}$ et $\phi_{0}$ doit être satisfait quel que soit $\left(\theta_{0}, \phi_{0}\right)$.

$\mathrm{Si}$ et seulement si, ces deux conditions sont réalisées, on peut écrire :

$$
\left[\begin{array}{c}
X_{0}^{2} \\
Y_{0}^{2} \\
Z_{0}^{2}
\end{array}\right]=\left[\begin{array}{ccc}
\frac{1}{M_{1}^{\prime 2}} & & \\
& \frac{1}{M_{2}^{\prime 2}} & \\
& & \frac{1}{M_{3}^{\prime 2}}
\end{array}\right]\left[\begin{array}{l}
m_{\mathrm{r}}^{\prime 2} X^{\prime 2} \\
m_{\mathrm{r}}^{\prime 2} Y^{\prime 2} \\
m_{\mathrm{r}}^{\prime 2} Z^{\prime 2}
\end{array}\right]
$$

Le modèle transforme la sphère de rayon unité, indicatrice de la direction du champ magnétique dans le milieu non déformé en un ellipsoïde qui est l'indicatrice de l'aimantation rémanente dans le milieu déformé.

On a :

$$
\begin{aligned}
& \frac{1}{M_{1}^{\prime 2}}=\left(a_{00}-a_{20}\right)+2 a_{22} \\
& \frac{1}{M_{2}^{\prime 2}}=\left(a_{00}-a_{20}\right)-2 a_{22} \\
& \frac{1}{M_{3}^{\prime 2}}=\left(a_{00}+a_{20}\right) .
\end{aligned}
$$

L'assimilation du modèle à une transformation linéaire implique une approximation sur la forme de la surface indicatrice de $\mathbf{m}_{\mathrm{r}}$ et sur la direction de $\mathbf{m}_{\mathbf{r}}$.

Deuxième partie : application du modèle à un cas particulier.

\section{Etude détaillée d'un cas concret : déformation coaxiale de révolution isovolumique. Aplatissement ou étirement pur.}

Cest le cas le plus simple que l'on puisse envisager. Le problème est indépendant de $\phi$ et de révolution autour de $O Z$. Il suffit de l'étudier dans un plan contenant cet axe par exemple $Z O X$. Les symétries $\mathrm{du}$ problème permettent de restreindre l'étude aux seules valeurs de $\theta_{0}$ comprises entre 0 et $\pi / 2$. Les marqueurs sont allongés, linéaires à la limite $(\chi=1)$. Le paramètre du problème est : $k^{\prime}=$ $\lambda_{3}^{\chi}$; dans le cas d'un marqueur de March $k^{\prime}=$ $\lambda_{3}$.

Nous nous bornerons aux deux processus d'aimantation : grains fins et polydomaines idéaux. Ce qui 
revient à dire que nous considérons les deux extrêmes d'une large granulométrie des marqueurs. Pour le premier type de grains on peut se borner à $\chi=1$, on se ramène au cas $\chi \neq 1$ à l'aide du "critère de similitude ». A un rapport d'axes $R_{1}=c_{1} / a_{1}$ correspond $\lambda_{1}$ et une valeur $k_{1}^{\prime}$ du paramètre du problème. L'anisotropie magnétique cherchée correspond à $k_{1}^{\prime}$. Il n'en est plus de même dans le cas du polydomaine idéal.

\subsection{LES INDICATRICES ET LEUR ÉVOLUTION.}

5.1.1 Aimantation rémanente relative dans un processus de grains fins : ARIS, ATR, ARISD, ATRD. Nous distinguerons d'emblée aplatissement et étirement. La figure 1 donne schématiquement l'allure des indicatrices et de leur évolution pour une déformation croissante. Celles-ci sont voisines de quarts d'ellipse, analogues à ceux caractérisant la déformation finie. L'anisotropie magnétique a la symétrie cylindrique. Pour un aplatissement le plan $X \mathrm{O} Y$ est un plan de facile aimantation rémanente avec isotropie dans ce plan. L'axe $\mathrm{O} Z$ est un axe de difficile aimantation rémanente. Pour l'étirement $\mathrm{O} Z$ est un axe facile alors que $X O Y$ est un plan de difficile aimantation rémanente. Cette dernière est isotrope dans ce plan.

Les figures Ia, Ib montrent l'allure et les positions respectives des trois indicatrices ARIS-ARISD, ATR, ATRD ainsi que leur évolution en fonction du paramètre $k^{\prime}=\lambda_{3}^{\chi}=\lambda_{3}$. L'axe de facile aimantation rémanente correspond à l'axe d'allongement. La surface indicatrice, rapportée aux axes principaux de déformation, est qualitativement semblable à l'ellipsoïde de déformation finie.

Les figures Ic, Id regroupent l'évolution de l'indicatrice des grandeurs non déformées.

5.1.2 Susceptibilité relative en champ faible d'un polydomaine idéal, déterminée suivant la direction du champ magnétisant. - La figure 2 montre schématiquement l'allure des indicatrices et leur évolution pour une déformation croissante. Il s'agit là de l'aspect habituel des courbes de susceptibilité. L'anisotropie magnétique correspondante est caractérisée pour $\lambda_{3}<1$ par une indicatrice ayant un plan
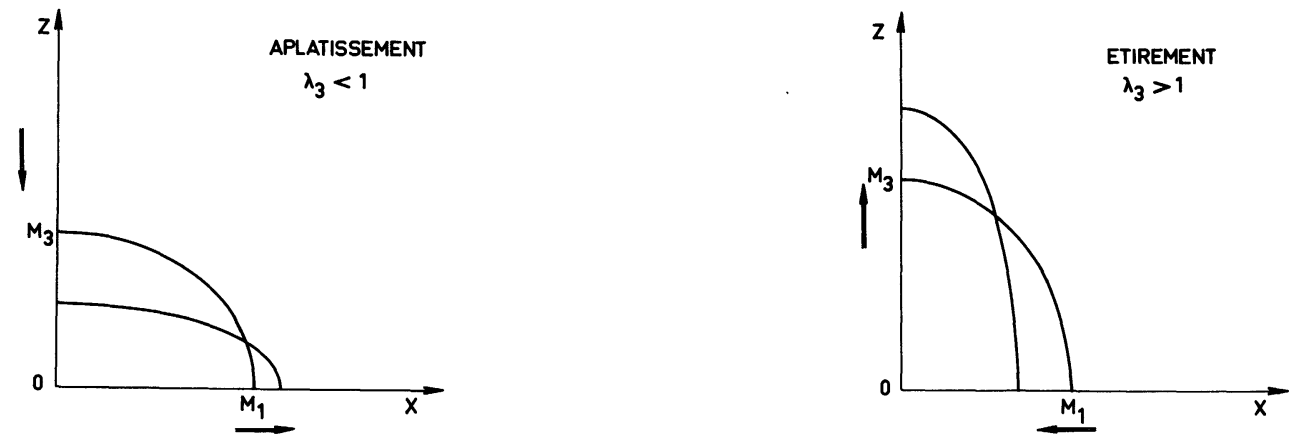

Fig. 1. - Allure des indicatrices de l'aimantation rémanente. Les flèches indiquent la façon dont l'indicatrice évolue quand la déformation finie croît.

[Shape of the indicatric of the remanent magnetization. The arrows show the sense of evolution as the finite deformation increases.]
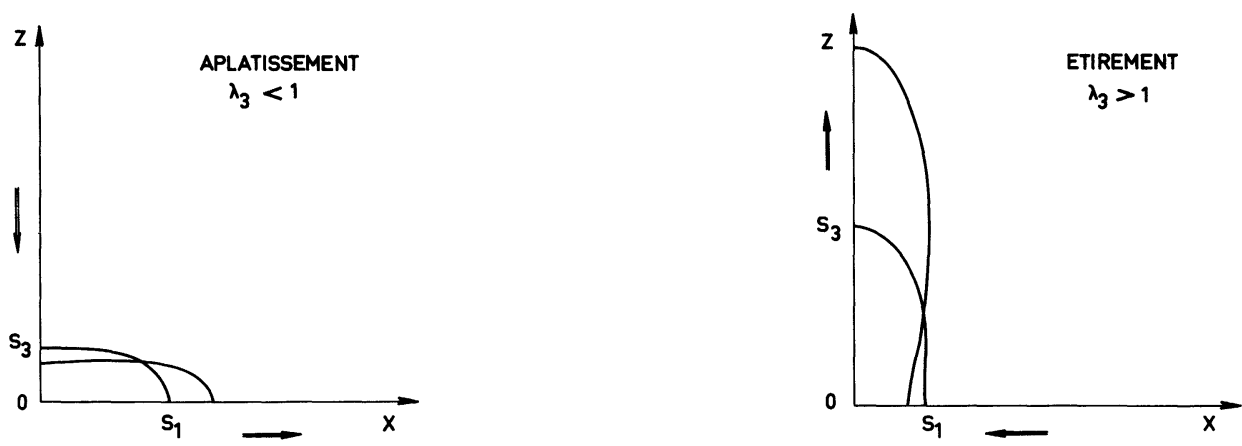

Fig. 2. - Allure des indicatrices de susceptibilité relative. Les flèches indiquent la façon dont l'indicatrice évolue quand la déformation finie croît.

[Shape of the indicatric of the relative susceptibility. The arrows show the sense of evolution as the finite deformation increases.] 

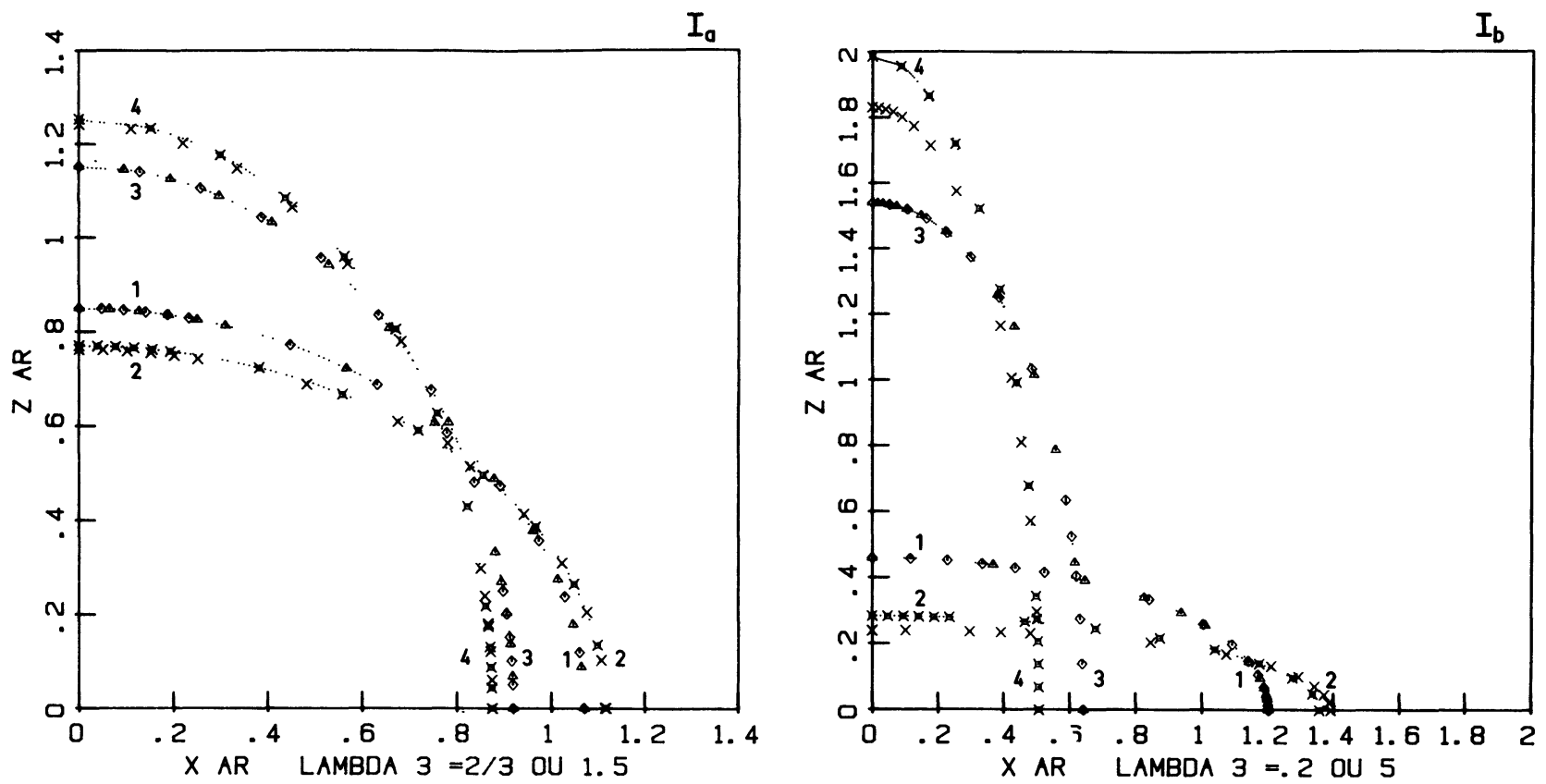

Figs. Ia, Ib. - Les indicatrices de l'aimantation rémanente, leurs positions respectives et leur évolution en fonction de la déformation finie. Aplatissement : (1) ARIS-ARISD ; (2) ATR ; (X) ATRD. Etirement : (3) ARIS-ARISD ; (4) ATR. (X) ATRD.

[Indicatrices of the remanent magnetization, their respective positions and evolutions with finite deformation. Flattening: (1) saturated isothermal remanent magnetization (SIRM) - deformed saturated isothermal remanent magnetization (DSIRM) ; (2) thermoremanent magnetization TRM ; (X) deformed thermoremanent magnetization DTRM. Stretching : (3) SIRM-DSIRM ; (4) TRM ; (X) DTRM.]
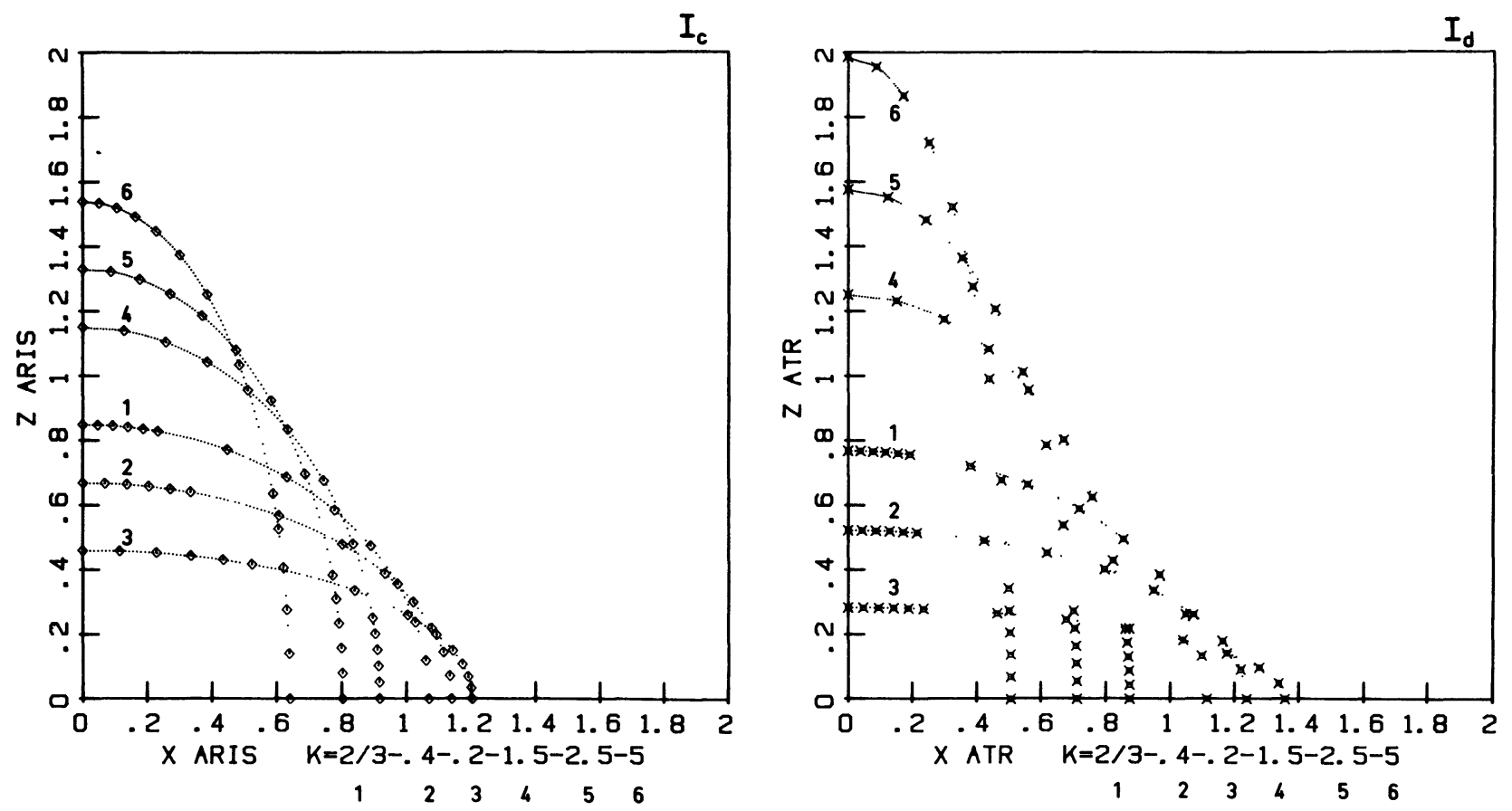

Figs. Ic, Id. - Evolution des indicatrices de l'ARIS (Ic) et de l'ATR (Id) pour des valeurs croissantes de la déformation finie. Aplatissement : 1, 2, 3. Etirement : 4, 5, 6.

[Evolution of indicatrices of the SIRM (Ic) and the TRM (Id) for increasing finite deformations. Flattening : $1,2,3$. Stretching : $4,5,6$.] 

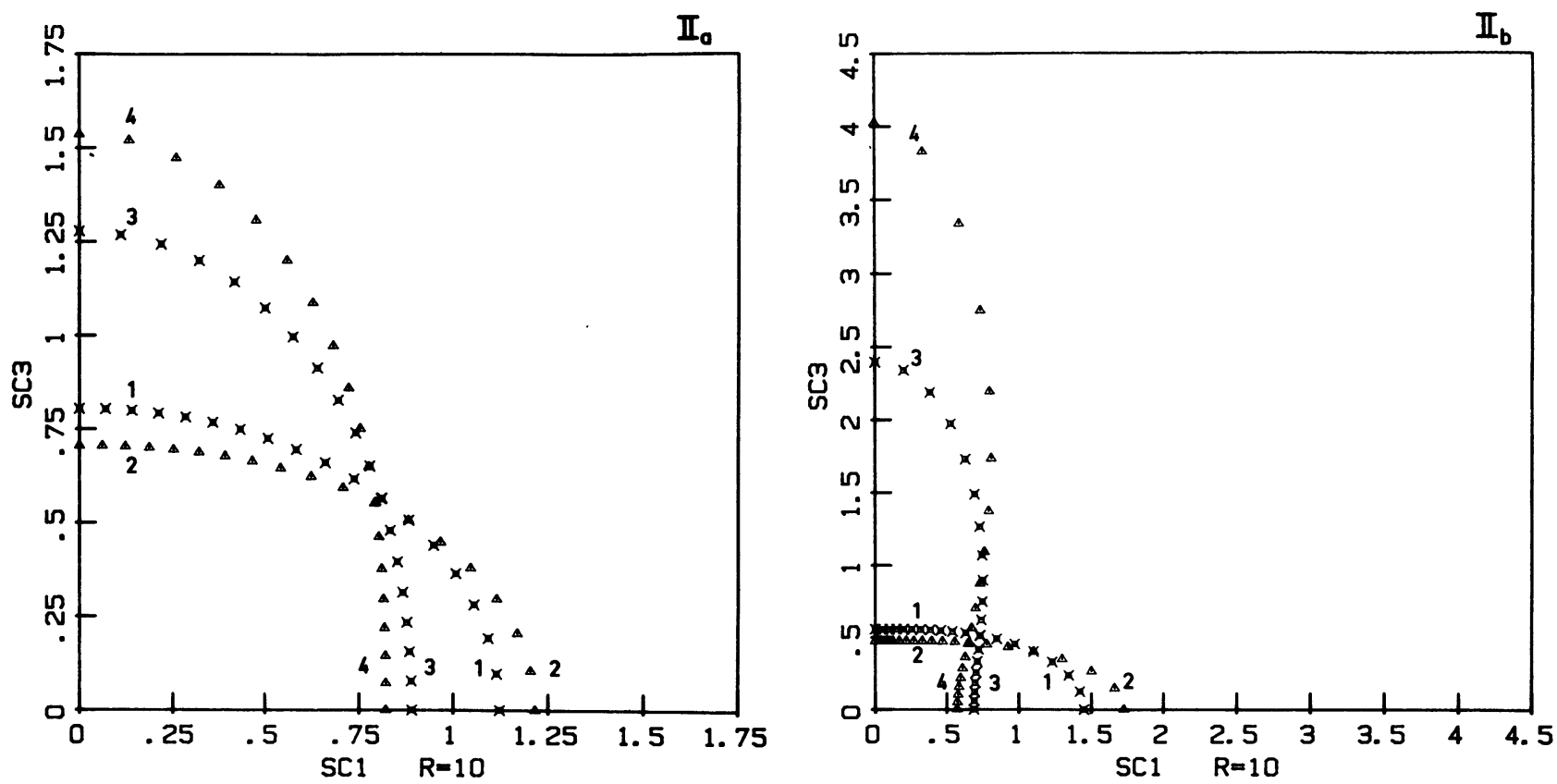

Figs. IIa, IIb. - Allure des indicatrices de susceptibilité relative dans le plan $Z O X$; leur évolution en fonction de la déformation finie à $R$ constant. IIa : Aplatissement : (1) $\lambda_{3}=2 / 3$; (2) $\lambda_{3}=0,5$. Etirement : (3) $\lambda_{3}=1,5 ;(4)$ $\lambda_{3}=2$. IIb : Aplatissement : (1) $\lambda_{3}=0,25$; (2) $\lambda_{3}=0,1$. Etirement : (3) $\lambda_{3}=4$; (4) $\lambda_{3}=10$.

[Shape of the indicatric of relative susceptibility in the $Z O X$ plane, and evolution as a function of the finite deformation at constant $R$. IIa : Flattening : (1) $\lambda_{3}=2 / 3$; (2) $\lambda_{3}=0.5$. Stretching : (3) $\lambda_{3}=1.5 ;$ (4) $\lambda_{3}=2$. IIb : Flattening : (1) $\lambda_{3}=0.25$; (2) $\lambda_{3}=0.1$. Stretching: (3) $\lambda_{3}=4$; (4) $\lambda_{3}=10$.]
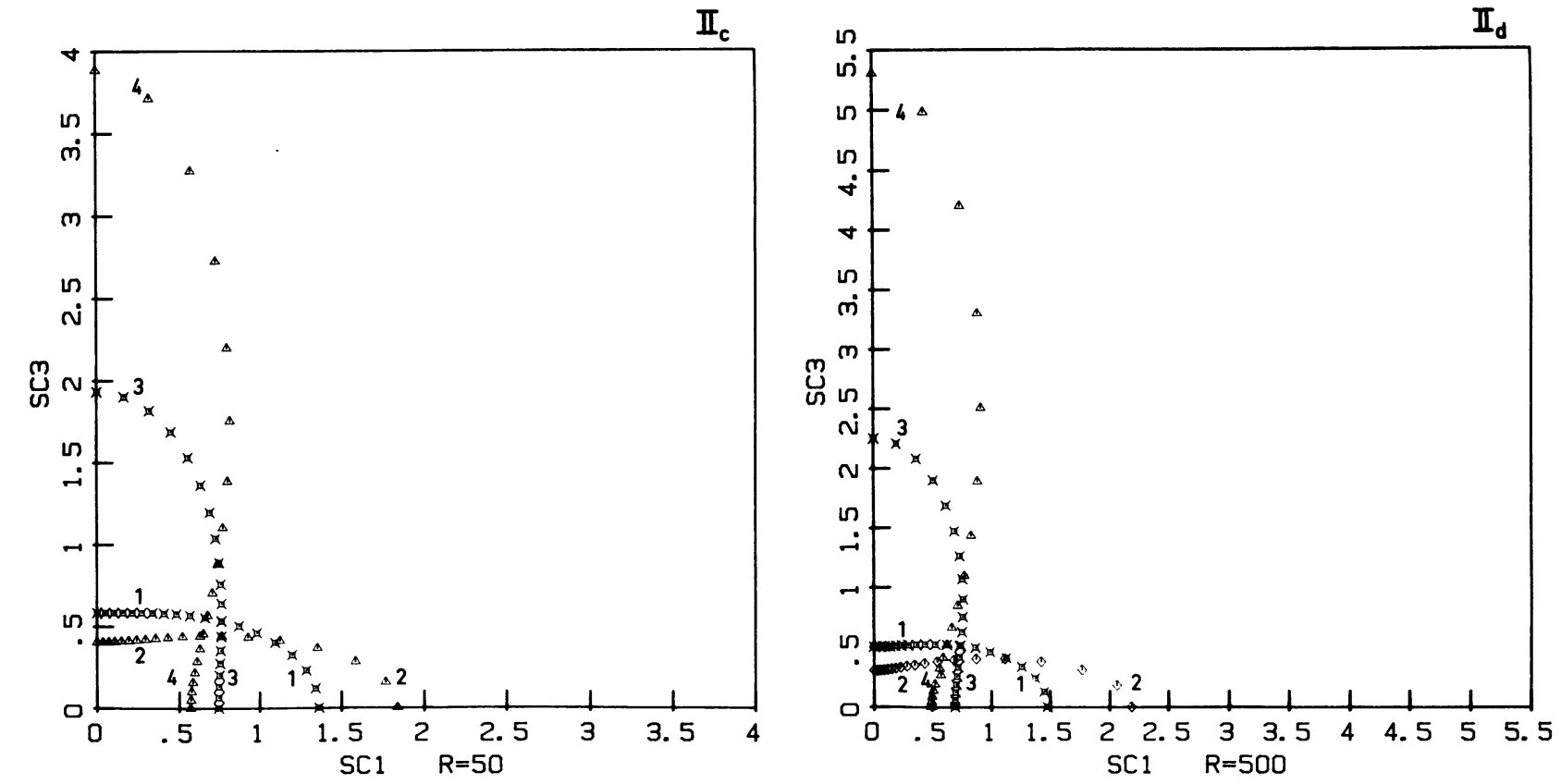

Figs. IIc, IId. - Evolution de l'allure des indicatrices de susceptibilité relative en fonction de $R$. IIc: $R=$ 50 ; (1) $\lambda_{3}=0,5$; (2) $\lambda_{3}=0,25$; (3) $\lambda_{3}=2$; (4) $\lambda_{3}=4$. IId : $R=500$. Mêmes notations et valeurs de $\lambda_{3}$.

[Evolution of the indicatrices of the relative susceptibility with $R$. IIc : $R=50$; (1) $\lambda_{3}=0.5 ;(2) \lambda_{3}=0.25$; $\lambda_{3}=2 ;$ (4) $\lambda_{3}=4$. IId : $R=500$, same symbols and values of $\lambda_{3}$.] 
de susceptibilité isotrope maximale $X O Y\left(S_{1}=\right.$ $S_{2}$ ) et un axe de susceptibilité minimale $\mathrm{OZ}$ : $\left(S_{3}<S_{1}=S_{2}\right)$.

Pour $\lambda_{3}>1$, on a au contraire un axe $\mathrm{OZ}$ de plus grande susceptibilité $\left(S_{3}>S_{1}=S_{2}\right)$ et un plan $X O Y$ de susceptibilité minimale isotrope $\left(S_{1}=S_{2}\right)$.

Les figures IIa, IIb montrent la forme de l'indicatrice, rapportée aux axes principaux de déformation, dans le cas d'un aplatissement ou d'un étirement, pour des marqueurs de rapport d'axes $R=c / a=$ 10. Elles montrent également son évolution en fonction de l'intensité de la déformation caractérisée par $\lambda_{3}$.

Les figures IIc, IId illustrent l'évolution de l'indicatrice en fonction de $R$. Celle-ci sera chiffrée par la suite lors des développements en série de Fourier (cf. Tab. VI). La déformation de l'indicatrice quand $R$ croît se traduit par une importance croissante dans le développement des termes d'ordre supérieur à deux.

Le modèle de March correspond à $R \infty$. On ne peut, pour des raisons de divergence, faire sans précautions $N_{3}=0$ dans l'expression de la susceptibilité. Par contre cette dernière reste finie pour des valeurs de $\chi$ aussi proches de 1 qu'on le désire.

$R=500$ correspond à $\chi=0,999992$ soit à $8 \times$ $10^{-6}$ près à la valeur 1 . Nous considérerons que $R=500$ représente le modèle de March.

\subsection{COMPARAISON DE L'ANISOTROPIE DES AIMAN-} TATIONS RÉMANENTES ET DE LA SUSCEPTIBILITÉ RELATIVE. - Nous avons envisagé deux processus d'aimantation limite pour les grains. Les grandeurs physiques pertinentes associées à ces mécanismes sont : les aimantations rémanentes et la susceptibilité en champ faible. La cause de l'anisotropie magnétique étant dans les deux cas la déformation finie, un modèle cohérent doit conduire à des résultats semblables pour l'anisotropie des grandeurs pertinentes. C'est bien ce que l'on observe.

5.2.1 Comparaison des indicatrices. - L'indicatrice de la composante de l'aimantation rémanente suivant la direction du champ magnétisant est qualitativement comparable à celle de la susceptibilité (cf. Figs. IIIa, IIIb). Il en est de même de son évolution en fonction de la déformation. Les figures IIIa' et $^{\prime}$ III' ' montrent l'allure des composantes de l'aimantation rémanente dans la direction perpendiculaire au champ.

5.2.2 Comparaison de l'évolution de $a_{\mathrm{m}}, a_{\mathrm{d}} ; a_{\mathrm{ms}}$, $a_{\mathrm{d}}$ en fonction de $\lambda_{3}$ et de $\varepsilon_{3}$. - Nous définirons $a_{\mathrm{m}}$ et $a_{\mathrm{ms}}$ comme étant le rapport $M_{1} / M_{3}$ et $S_{1} / S_{3}$ des aimantations rémanentes et des susceptibilités principales suivant $\mathrm{O} X$ et $\mathrm{OZ}$. L'anisotropie mécanique $a_{\mathrm{d}}$ de la déformée est égale au rapport des demi-grands axes de l'ellipse de déformation finie dans le plan $Z O X$ :

$$
a_{\mathrm{d}}=\left(\frac{\lambda_{1}}{\lambda_{3}}\right)^{1 / 2} ;
$$

$a_{\mathrm{d}}$ vaut ici $\lambda_{3}^{-3 / 4}$.

Evolution en fonction de $\lambda_{3}$ (Figs. IVa, $\mathrm{a}^{\prime} ; \mathrm{IVb}$, $\left.b^{\prime}\right)$

Les évolutions de $a_{\mathrm{m}}$ (Figs. IVa, b) et $a_{\mathrm{ms}}$ (Figs. IVa', b') sont qualitativement comparables avec des ordres de grandeur qui le sont également. $a_{\mathrm{ms}}$ est d'autant plus grand que la déformation est importante et que $R$ est grand.

$\left.\mathrm{d}^{\prime}\right)$.

Evolution en fonction de $\varepsilon_{3}$ (Figs. IVc, $c^{\prime}$; IVd,

La déformation naturelle est le paramètre physique du problème. Le tracé des graphes Log-Log montre dans les deux cas des départs linéaires à partir de l'état initial isotrope non déformé

Dans les deux cas on a, dans cette partie de la courbe, une loi du type :

$$
\begin{aligned}
a_{\mathrm{m}} & =\exp \left(-2 m \varepsilon_{3}\right) \\
a_{\mathrm{ms}} & =\exp \left(-2 m^{\prime} \varepsilon_{3}\right) .
\end{aligned}
$$

La limite de validité de cette loi est du même ordre dans les deux cas.

5.2.3 Dissymétrie entre aplatissement et étirement. $\mathrm{Si}$ à la valeur $\lambda_{3}=1 / n$ correspond une anisotropie $a_{\mathrm{d}}$ de la déformée, pour la valeur $\lambda_{3}=n$ on a $1 / a_{\mathrm{d}}$. Cette propriété n'est plus valable pour $a_{\mathrm{m}}$ et $a_{\mathrm{ms}}$.

Les courbes $a_{\mathrm{m}}$ et $a_{\mathrm{ms}}$ en fonction de $\lambda_{3}$ ont un point anguleux qui n'est autre que celui représentant l'état isotrope initial $(1,1)$ (cf. Figs. IVa, IVb, IVa', $\mathrm{IVb}^{\prime}$ ).

Il est à remarquer qu'on a d'autres exemples où les courbes représentatives de certaines propriétés magnétiques ont un point anguleux au passage traction, compression [13].

Les courbes $a_{\mathrm{m}}=f\left(a_{\mathrm{d}}\right)$ pour $\lambda_{3}<1$ et $1 / a_{\mathrm{m}}=$ $g\left(1 / a_{\mathrm{d}}\right)$ pour $\lambda_{3}>1$ et leur pendant pour $a_{\mathrm{ms}}$ montrent bien la différence de comportement entre les deux cas (cf. Figs. $\mathrm{Va}, \mathrm{Vb} ; \mathrm{Va}^{\prime}, \mathrm{Vb}^{\prime}$ ). Comme de plus elles sont situées de part et d'autre de la première bissectrice il en résulte qu'il n'y a pas identité entre fabrique magnétique et mécanique.

\subsection{RÉSULTATS SPÉCIFIQUES RELATIFS A L'AIMAN- TATION RÉMANENTE ET À LA SUSCEPTIBILITÉ}

5.3.1 Evolution des déphasages; leur intérêt. Nous avons défini le déphasage comme étant l'angle $\Delta$ entre la direction du champ magnétisant et de l'aimantation rémanente.

Les figures VI et VII montrent respectivement, pour les diverses grandeurs étudiées, l'évolution de $\Delta$ :

- en fonction de $\theta_{0}$ avec $k^{\prime}=\lambda_{3}$ en paramètre (Figs. VIa, b, c, d) 

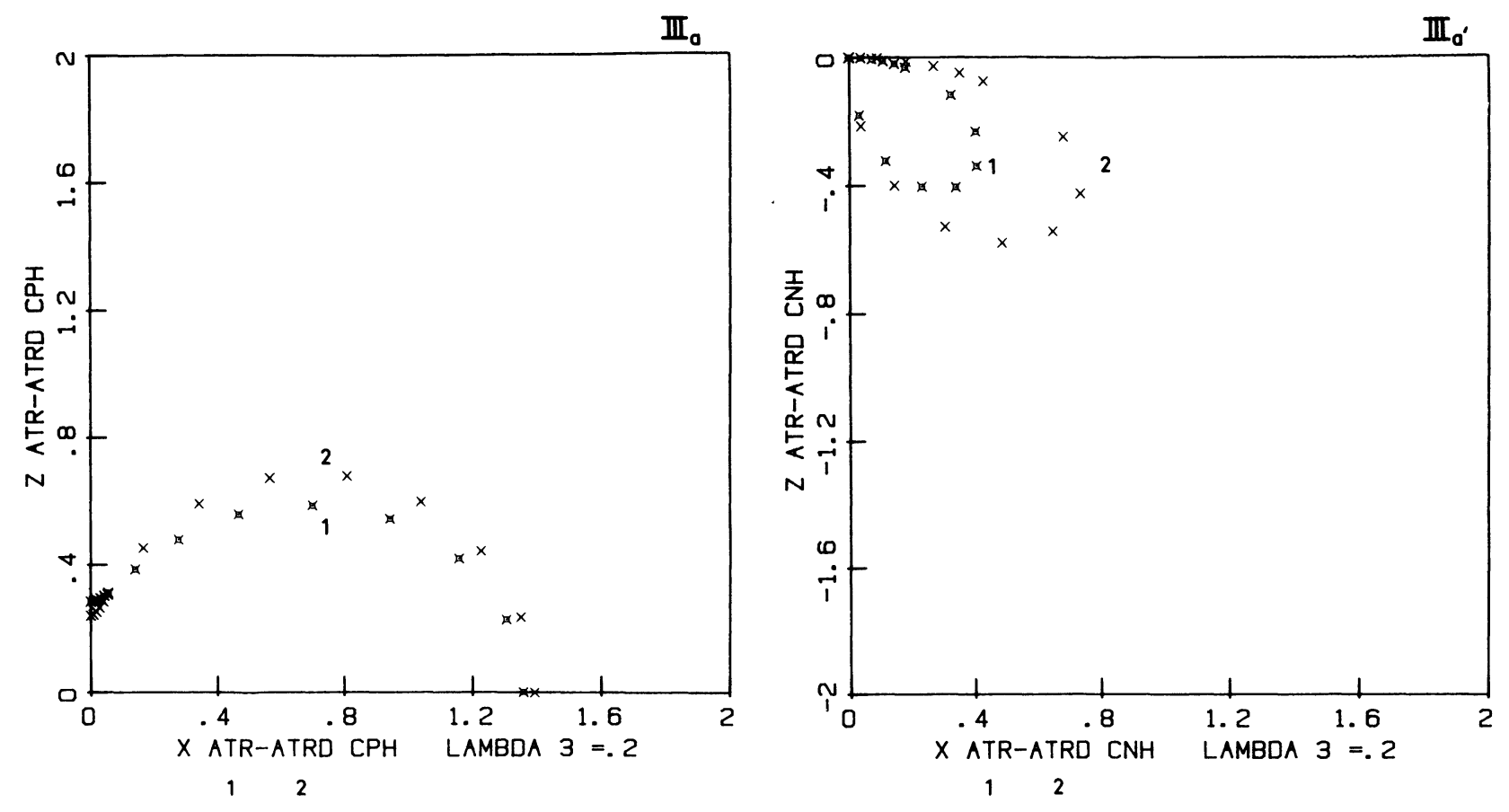

Figs. IIIa, IIIa'. - Cas de l'aplatissement $\left(\lambda_{3}=0,2\right)$. Allure des composantes de l'ATR (1) et de l'ATRD (2). IIIa : Composantes suivant la direction du champ magnétisant : CPH. IIIa' : Composantes suivant la direction perpendiculaire au champ magnétisant. CNH.

[Case of flattening $\left(\lambda_{3}=0.2\right)$. Shape of the components of the TRM (1) and the DTRM (2). IIIa : Components along the magnetizating field : CPH. IIIa' : Components perpendicular to the magnetizing field : $\mathrm{CNH}$.]
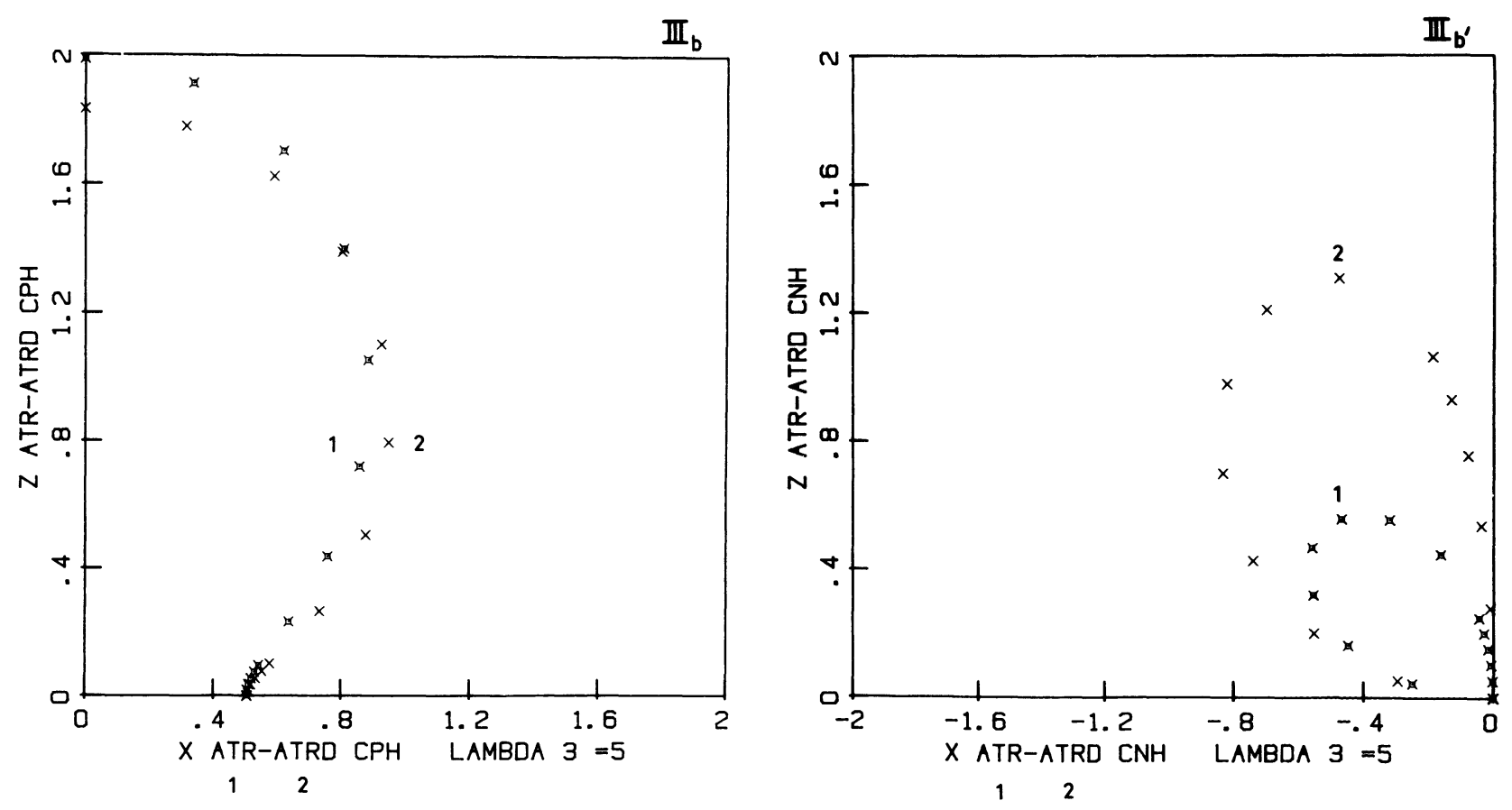

Figs. IIIb, IIIb'. - Cas de l'étirement $\left(\lambda_{3}=5\right)$. Mêmes notations que les deux figures précédentes.

[Case of stretching $\left(\lambda_{3}=5\right)$. Same symbols as the two preceding figures.] 

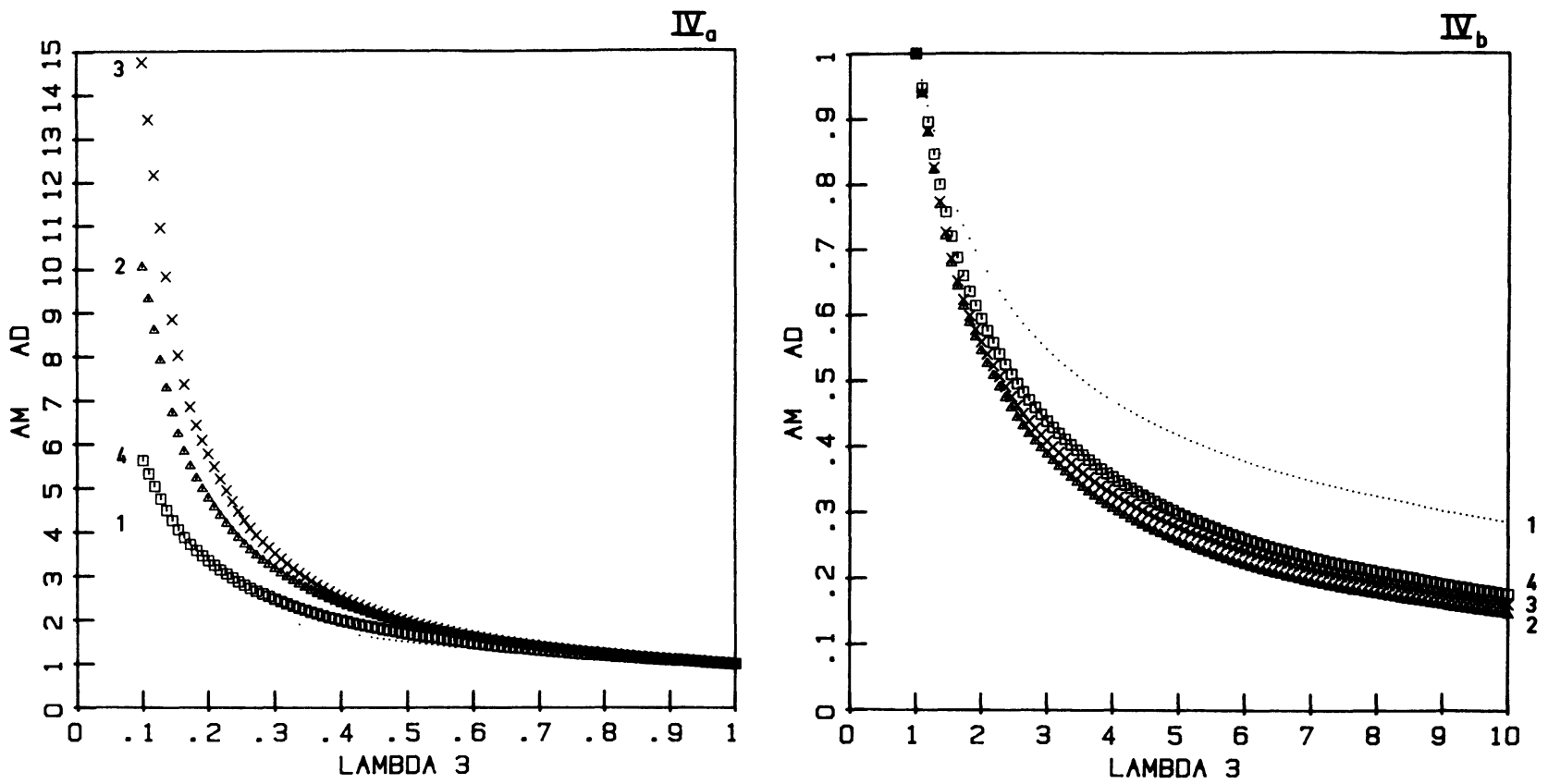

Figs. IVa, IVb. - Evolution de l'anisotropie de l'aimantation rémanente $(A M)$ et de l'anisotropie de la déformation finie $(A D)$ en fonction de $\lambda_{3}$. (1) ARIS-ARISD ; (2) ATR ; (3) ATRD ; (4) $A D$. IVa : aplatissement. IVb : étirement.

[Evolutions of the anisotropy of the remanent magnetization $(A M)$ and the anisotropy of the finite deformation $(A D)$ as functions of $\lambda_{3}$. (1) SIRM-DSIRM ; (2) TRM ; (3) DTRM ; (4) $A D$. IVa : flattening. IVb : stretching.]
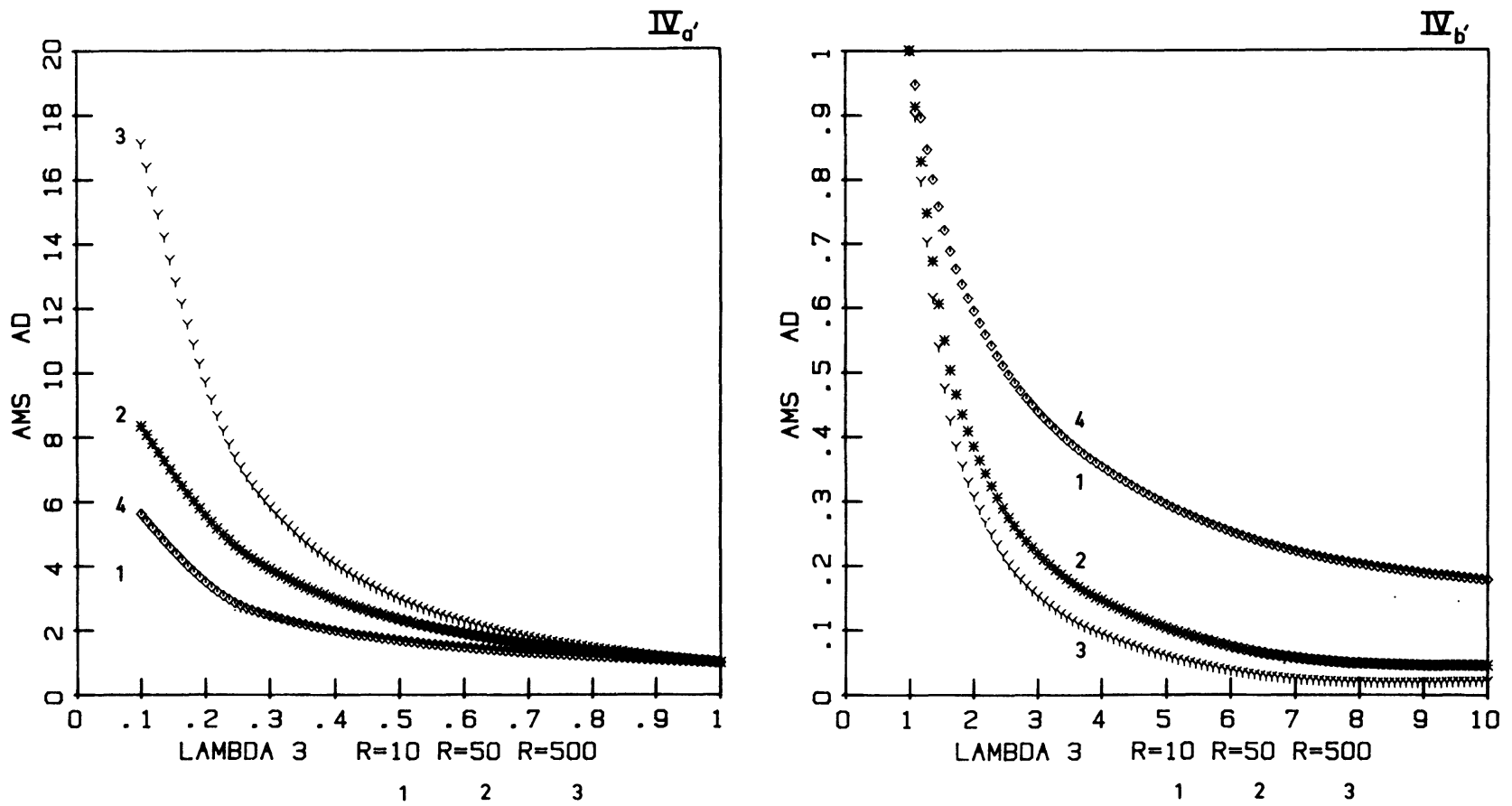

Figs. IVa', IVb'. - Evolution de l'anisotropie de susceptibilité relative $(A M S)$ en fonction de $\lambda_{3}$ pour diverses valeurs de $R$. Evolution de l'anisotropie de déformation finie $(A D)$ en fonction de $\lambda_{3}$. (1) $R=10$; (2) $R=50$; (3) $R=500$; (4) $A D$. IVa' : aplatissement. IVb' : étirement.

[Evolution of the anisotropy of the relative susceptibility $(A M S)$ with $\lambda_{3}$ for various values of $R$. Evolution of the anisotropy of the finite deformation with $\lambda_{3}$. (1) $R=10$; (2) $R=50$; (3) $R=500$; (4) $A D$. IVa' : flattening ; IVb': stretching.] 

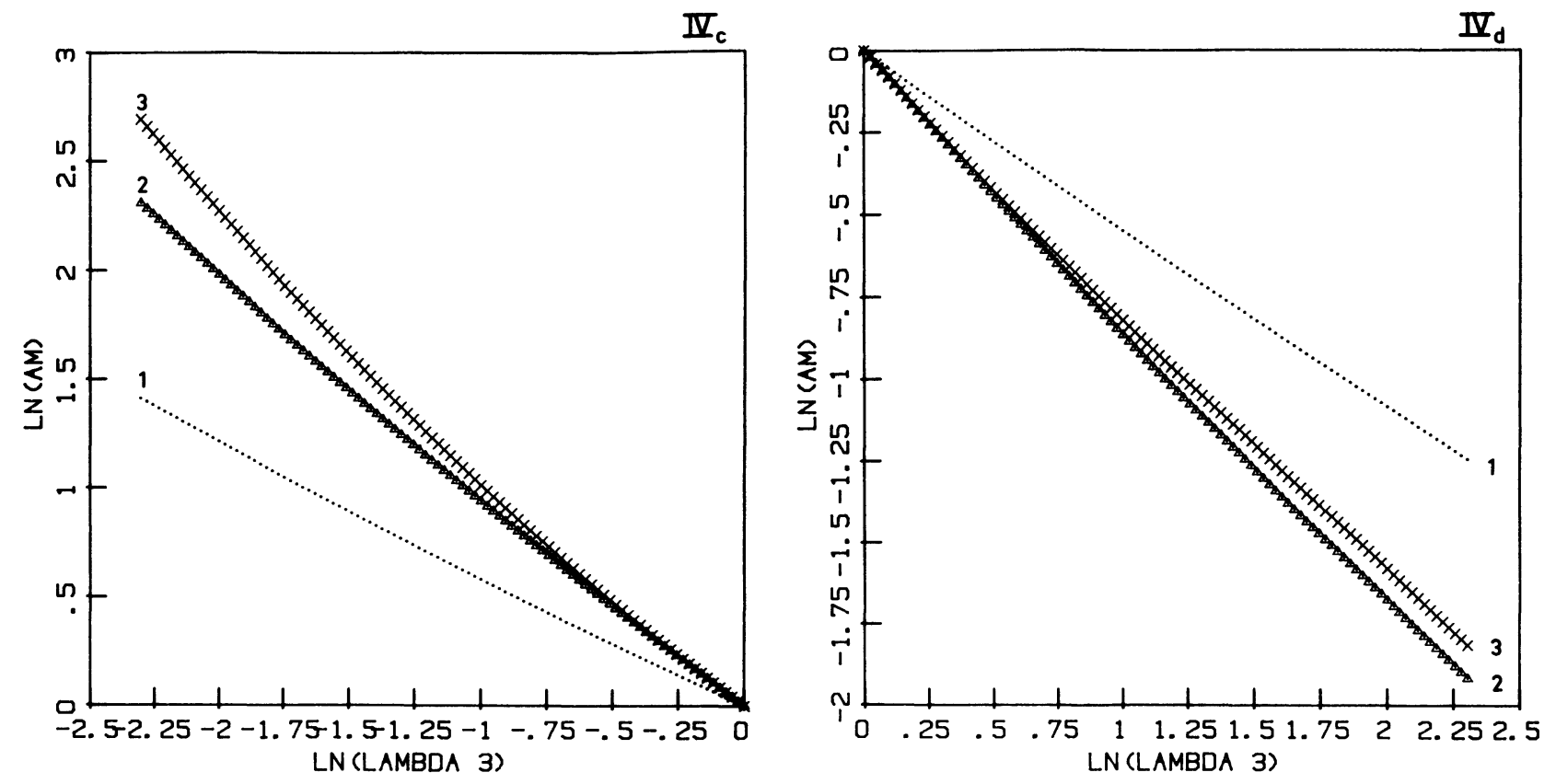

Figs. IVc, IVd. - Evolution du logarithme de l'anisotropie de l'aimantation rémanente en fonction de la déformation naturelle ( $\ln \lambda_{3}=2 \varepsilon_{3}$ ). (1) ARIS-ARISD ; (2) ATR ; (3) ATRD. IVc : aplatissement ; IVd : étirement.

[The anisotropy of the remanent magnetization versus the natural deformation (logarithmic plot, $\ln \lambda=2$ ). (1) SIRMDSIRM ; (2) TRM ; (3) DTRM. IVc : flattening ; IVd : stretching.]
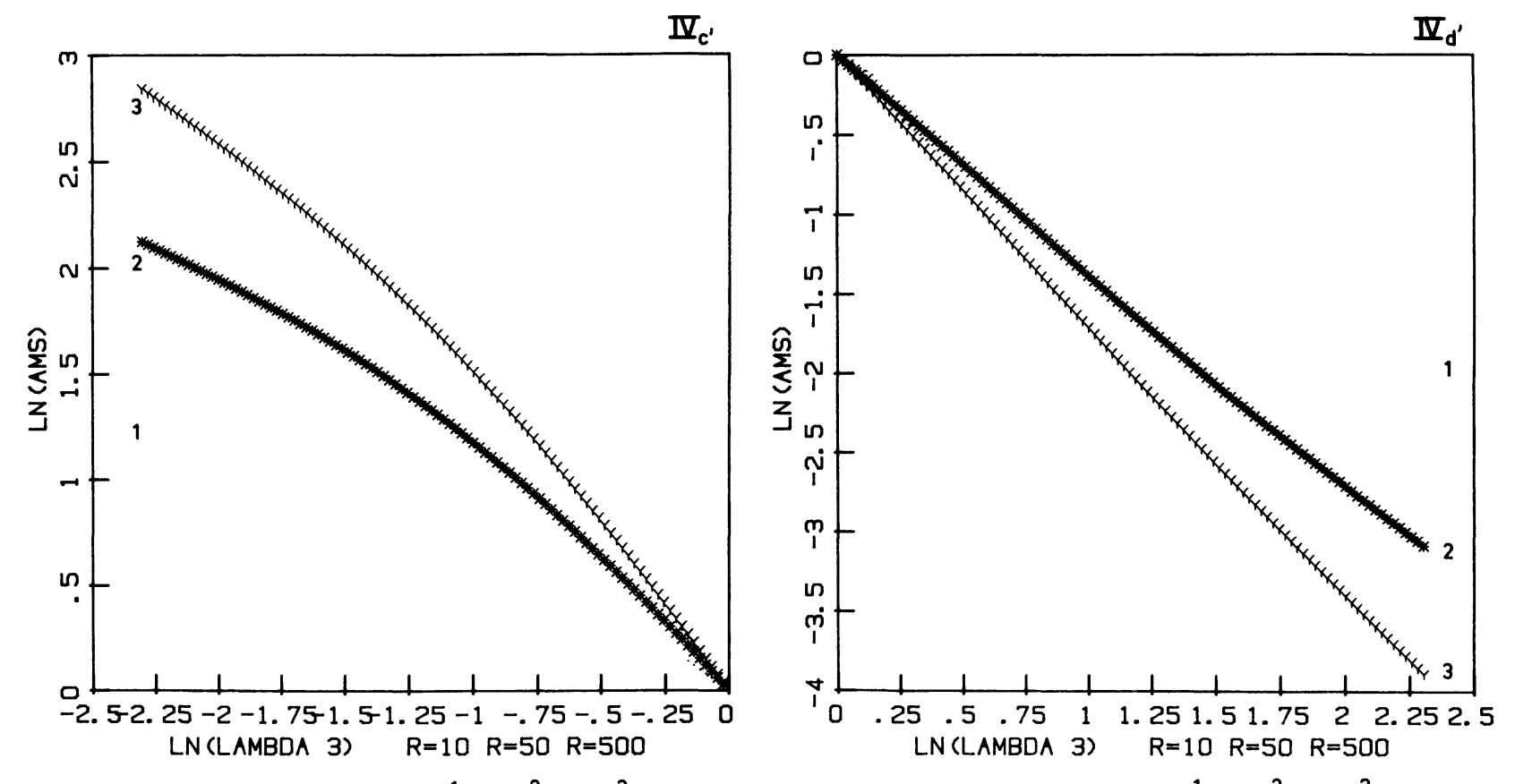

Figs. IVc', IVd'. - Evolution du logarithme de l'anisotropie de susceptibilité relative à $R$ donné en fonction de la déformation naturelle $\left(\operatorname{Ln} \lambda_{3}=2 \varepsilon_{3}\right)$. (1) $R=10$; (2) $R=50$; (3) $R=500$. IVc' : aplatissement ; IVd' : étirement.

[The anisotropy of the relative susceptibility at fixed $R$ versus the natural deformation (logarithmic plot, $\ln \lambda_{3}=2 \varepsilon$ ). (1) $R=10$; (2) $R=50$; (3) $R=500$. IVc' : flattening ; IVd' : stretching.] 

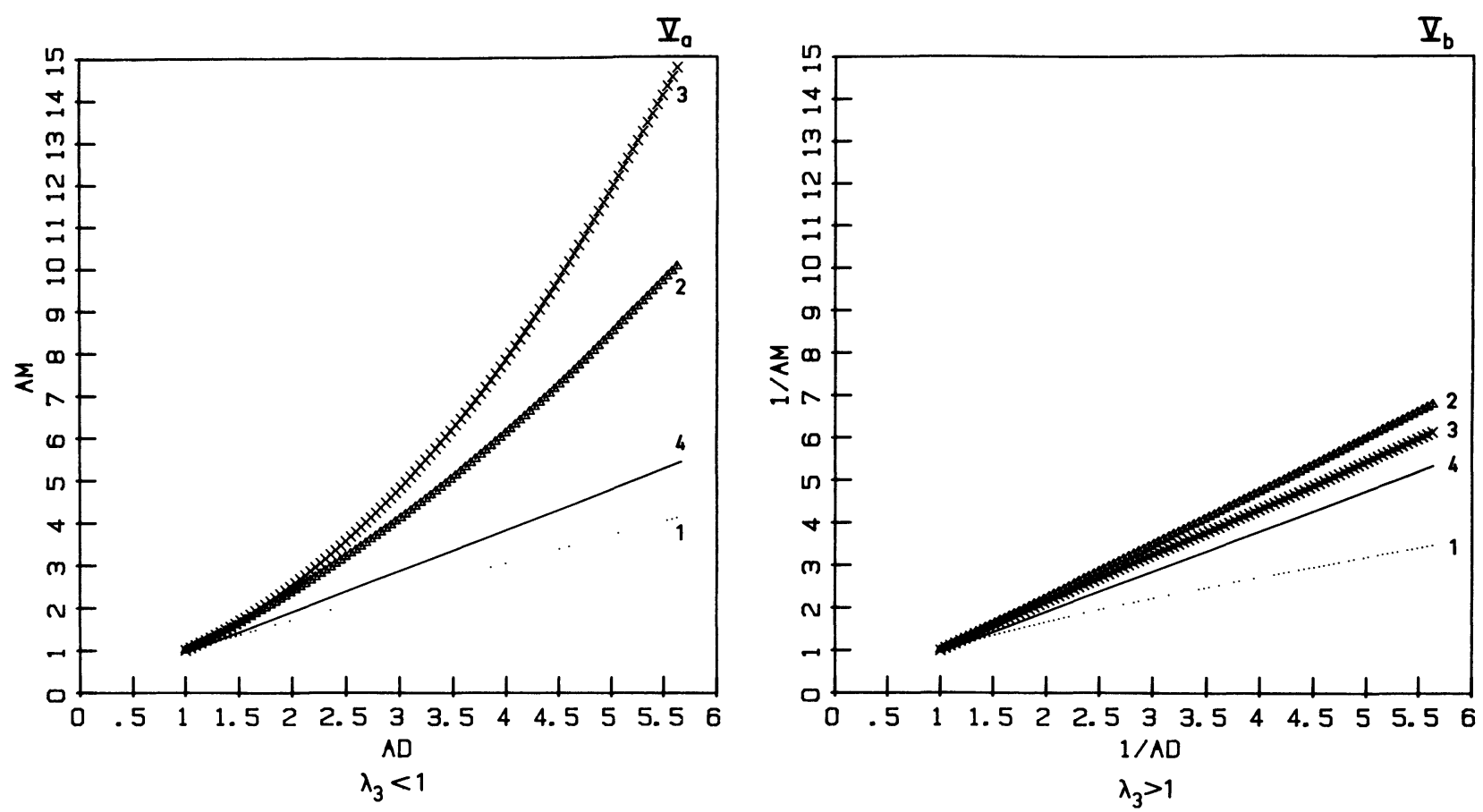

Figs. Va, Vb. - Dissymétrie entre aplatissement et étirement ; comparaison entre les graphes $A M=f(A D)(\mathrm{Va})$ et $1 / A M=g(1 / A D)(\mathrm{Vb})$. (1) ARIS-ARISD ; (2) ATR ; (3) ATRD. 4 représente la première bissectrice, soit le lieu des points ou $A M=A D$; c'est-à-dire l'identité des fabriques magnétique et mécanique.

[Non-symmetric effects of flattening and stretching; comparison between the graphs $A M=f(A D)$ (Va) and $1 / A M=g(1 / A D)(\mathrm{Vb})$. (1) SIRM-DSIRM ; (2) TRM ; (3) DTRM. 4 represents the first bisector, viz. the locus of points where $A M=A D$ : identical magnetic and mechanical fabrics.]
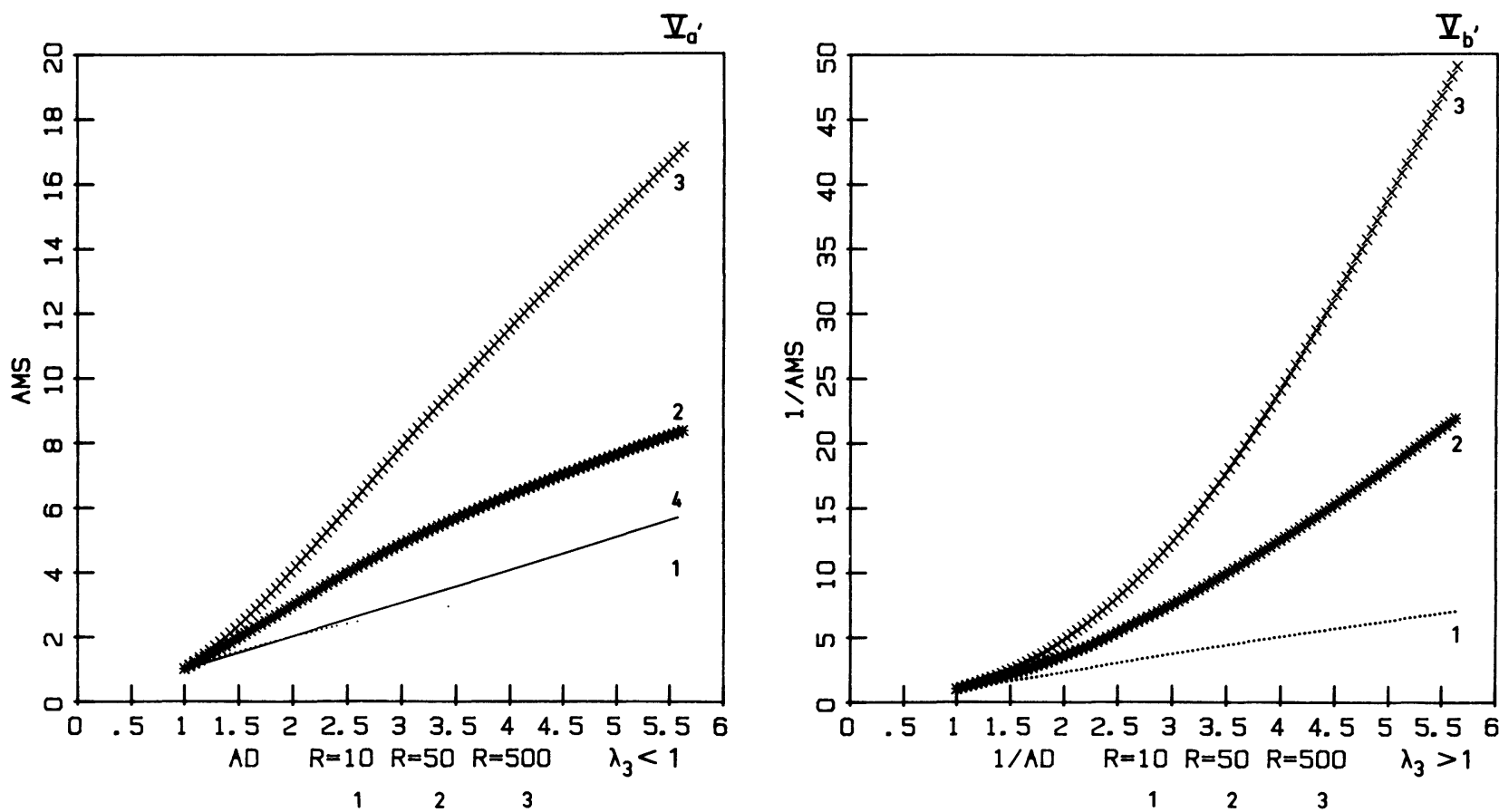

Figs. $\mathrm{Va}^{\prime}, \mathrm{Vb}^{\prime}$. - Dissymétrie entre aplatissement et étirement dans le cas de l'anisotropie de susceptibilité relative : comparaison des graphes $A M S=f^{\prime}(A D)\left(\mathrm{Va}^{\prime}\right)$ et $1 / A M S+g^{\prime}(1 / A D)\left(\mathrm{Vb}^{\prime}\right) .1: R=10 ; 2: R=50 ; 3:$ $R=500 ; 4:$ première bissectrice. Dans $(\mathrm{Vb})$ cette dernière est pratiquement confondue avec 1 .

[Non-symmetric effects of flattening and stretching on the anisotropy of the relative susceptibility ; comparison between the graphs $A M S=f^{\prime}(A D)\left(\mathrm{Va}^{\prime}\right)$ and $1 / A M S=g^{\prime}(1 / A D)\left(\mathrm{Vb}^{\prime}\right)$. (1) $R=10$; (2) $R=50$; (3) $R=500$; (4) first bisector; In $\mathrm{Vb}$, the latter pearly coincides with 1.$]$ 

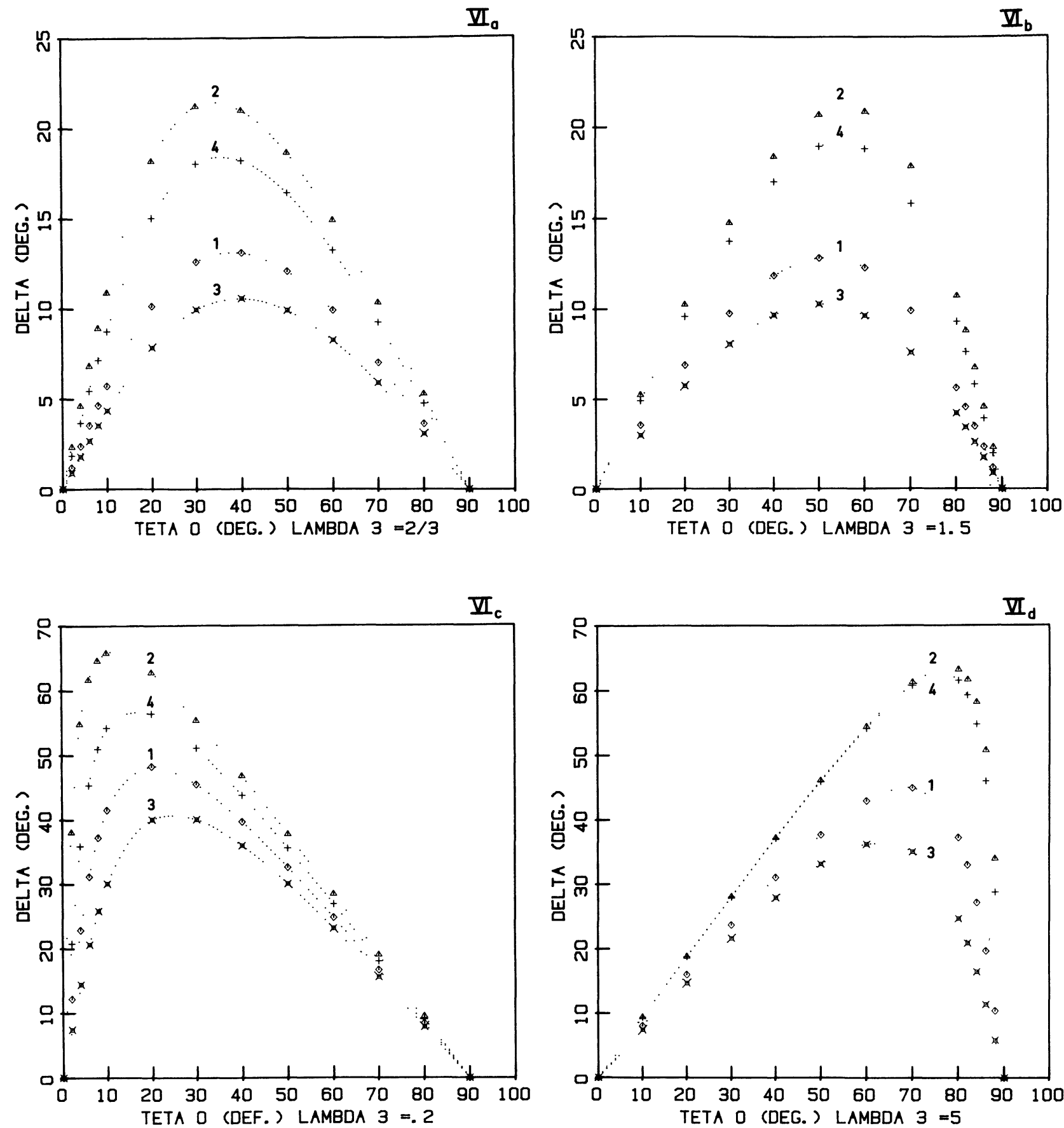

Figs. VIa, b, c, d. - Evolution de $\Delta$ en fonction de $\theta_{0}$ pour diverses valeurs de $\lambda_{3}$. Aplatissement : $\lambda_{3}=$ $2 / 3$ (VIa) ; $\lambda_{3}=0,2$ (VIc). Etirement : $\lambda_{3}=1,5$ (VIb) ; $\lambda_{3}=5$ (VId). ARIS (1) ; ARISD (2) ; ATR (3) ; ATRD (4).

[Evolution of $\Delta$ with $\theta_{0}$ for various values of $\lambda_{3}$. Flattening: $\lambda_{3}=2 / 3$ (VIa); $\lambda_{3}=0.2$ (VIc). Stretching : $\lambda_{3}=1.5$ (VIb) $; \lambda_{3}=5$ (VId). SIRM (1); DSIRM (2); TRM (3); DTRM (4).]

- en fonction de $\lambda_{3}$, ou de $1 / \lambda_{3}$ pour $\theta_{0}=$ $45^{\circ}$ (Figs. VIIa, b).

L'allure des courbes $\Delta=f\left(\theta_{0}\right)$ et leur évolution en fonction de la déformation s'interprète de façon très simple.

Pour un aplatissement $O Z$ est un axe de difficile aimantation et $X O Y$ un plan de facile aimantation. Si le champ magnétisant est suivant $O Z$ l'aimantation rémanente est également suivant cet axe : le déphasage est nul. Dès que le champ magnétisant s'écarte de $O Z$ l'aimantation rémanente se rapproche du plan facile et ce d'autant plus rapidement que la déformation est grande. $\Delta$ passe pour un maximum ; sa décroissance étant plus lente que n'avait été sa croissance.

Pour un étirement $\mathrm{O} Z$ est un axe de facile 

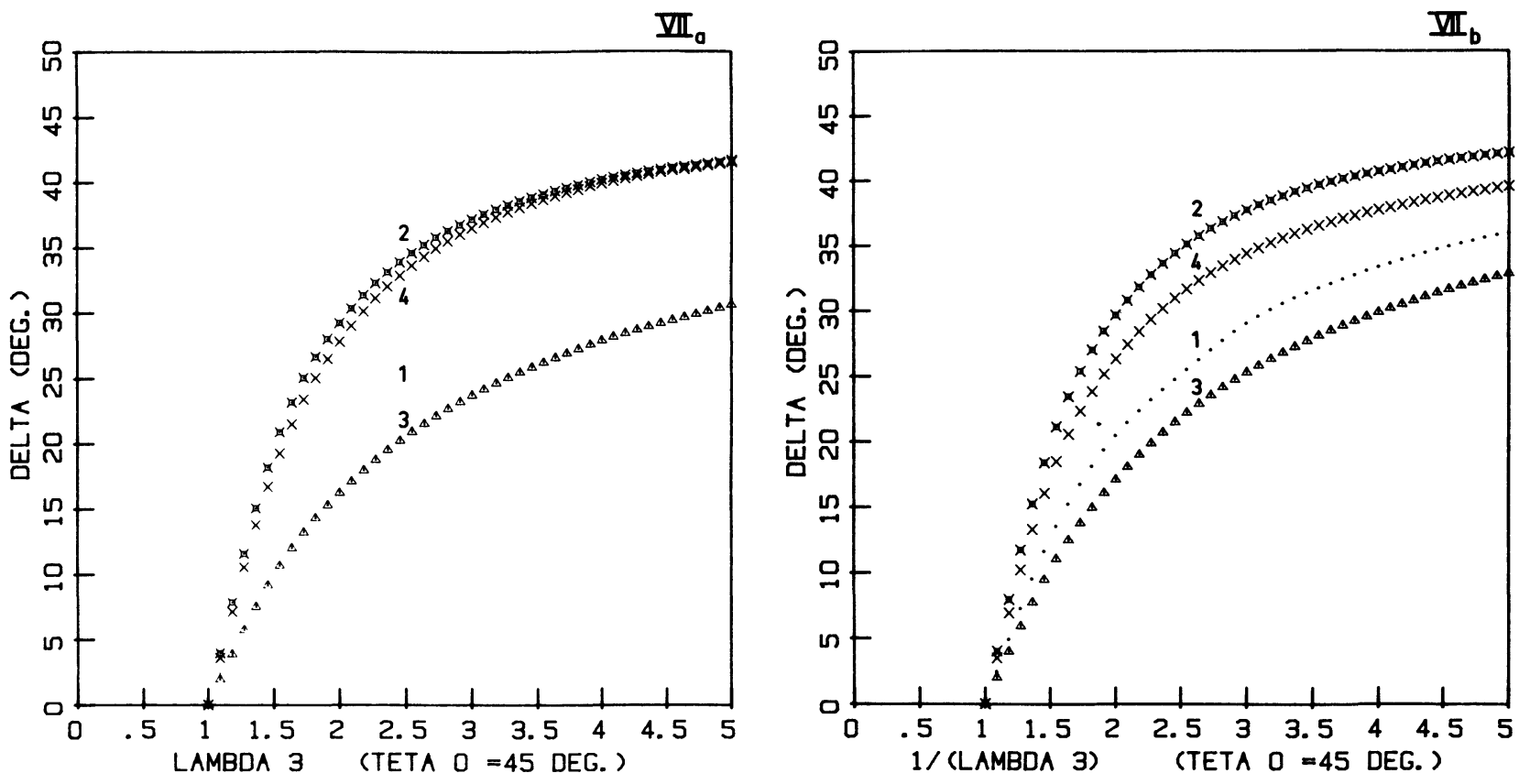

Figs. VIIa, VIIb. - Evolution de $\Delta$ à $\theta_{0}$ donné en fonction de $\lambda_{3}$ (aplatissement) ou de $1 / \lambda_{3}$ (étirement). ARIS (1) ; ARISD (2) ; ATR (3); ATRD (4).

[Evolution of $\Delta$ at fixed $\theta_{0}$ with $\lambda_{3}$ (flattening) or $1 / \lambda_{3}$ (stretching). SIRM (1) ; DSIRM (2) ; TRM (3) ; DTRM (4).]

aimantation et $X O Y$ un plan difficile. Quand la direction du champ magnétisant s'éloigne de $\mathrm{OZ}$ l'aimantation rémanente reste voisine de celui-ci ; $\Delta$ croît d'autant plus lentement que la déformation est plus grande, c'est-à-dire que l'axe est de plus facile aimantation. $\Delta$ passe par un maximum et décroît ensuite d'autant plus rapidement que la déformation est grande. L'aimantation rémanente bascule alors vers le plan de difficile aimantation.

Ces déphasages sont liés à la composante de l'aimantation rémanente perpendiculaire au champ. Les grandeurs déformées diffèrent essentiellement les grandeurs habituelles par la valeur de leurs composantes normales (cf. Figs. IIIa, a' ; IIIb, b').

Un des intérêts de l'étude des déphasages réside dans leur implication dans les problèmes de paléomagnétisme. On sait par des méthodes appropriées, à partir de l'aimantation rémanente naturelle, déterminer la direction du paléochamp qui en est l'origine. Mais quand on fait cette détermination on ne tient pas compte de la déformation interne de la roche. Ce point de vue n'est pas correct si la roche, aimantée au cours de sa formation a été tectonisée par la suite, cette déformation modifiant la direction de l'aimantation rémanente. On peut envisager une première approche du problème en procèdant de la manière suivante. On étudie d'abord l'anisotropie de l'ARIS de l'échantillon, ce qui permet de remonter à la déformation interne grâce au modèle. On détermine par ailleurs la direction de l'aimantation rémanente naturelle de l'échantillon. On sait de plus qu'il est souvent possible de considérer celle-ci comme une ATR. Le modèle permet alors connaissant la déformation interne de remonter au déphasage et a une valeur plus raisonnable de la direction du paléochamp (cf. Thèse Rochette [11]).

5.3.2 Analyse fine de la forme des indicatrices: développement en série de Fourier.

5.3.2.1 Comparaison entre les modèles de déformation finie et d'aimantation rémanente utilisés : similitude des fabriques mécanique et magnétique. $\overline{\text { Pour qu'il y ait similitude entre fabriques il faut que }}$ le modèle d'aimantation rémanente se réduise à une transformation linéaire.

Pour cela deux conditions sont nécessaires : (cf. 4.2)

- l'indicatrice doit être assimilable à une ellipse

- le caractère linéaire de la transformation impose une relation entre $\theta_{0}$ et $\theta_{1}$ qui doit être vérifiée quel que soit le couple $\theta_{0}, \theta_{1}$.

La première condition conduit à un développement en série de Fourier tel que l'ensemble des coefficients autres que $a_{0}$ et $a_{2}$ (terme constant et coefficient de $\cos 2 \theta$ ) soient négligeables. La seconde s'exprime par:

$$
\frac{\operatorname{tg} \theta_{0}}{\operatorname{tg} \theta_{1}}=\left(\frac{a_{0}-a_{2}}{a_{0}+a_{2}}\right)^{1 / 2}
$$

$\mathrm{Si}$ la première condition est seule remplie la similitude n'a lieu que suivant la direction des axes principaux qui correspondent à un déphasage nul.

Dans le cas où il y a similitude des fabriques le 
carré des aimantations rémanentes principales joue un rôle analogue aux $\lambda_{i}$.

Dans le modèle d'ATR développé, nous avons implicitement fait l'hypothèse d'un milieu linéaire en bornant le développement en série de la tangente hyperbolique au premier terme. Les opérations d'intégration conservent ce caractère de linéarité. Les résultats du calcul confirment bien ce point de vue. Les écarts observés ne sont pas significatifs et proviennent d'une précision insuffisante dans le calcul numérique des intégrales et des développements en série de Fourier. Le tableau II illustre ce qui précède.

Pour les trois autres grandeurs ARIS, ARISD, ATRD, il existe des éléments de non-linéarité qui font que seule la première condition est plus ou moins bien vérifiée. A toute direction $\theta_{0}$ du champ correspond une direction de l'aimantation rémanente $\theta_{1}$ qui n'est pas celle $\left(\theta_{1}\right)_{1}$ que l'on déduirait de la condition (2). Il en résulte une erreur sur le module et la direction de l'aimantation rémanente quand on fait l'approximation linéaire correspondant à $a_{0}$ et $a_{2}$. Les tableaux III et IV donnent les résultats de cette analyse dans le cas de l'ARIS pour $\lambda_{3}=$ $2,5, x=1$.

5.3.2.2 Aimantation rémanente parallèle et perpendiculaire au champ magnétisant ; caractère tensoriel de l'aimantation rémanente. - Dans le cadre du modèle est-il légitime d'écrire :

$$
\left(m_{\mathrm{r}}\right)_{i}=\left(M_{\mathrm{r}}\right)_{i j} \mathbf{h}_{j},
$$

$\mathbf{h}_{j}$ étant le vecteur unitaire caractérisant la direction du champ magnétisant. S'il n'en est pas ainsi, quelle approximation implique cette écriture ?

Sans le cas de la déformation coaxiale de révolution on $\mathrm{a}$ :

$$
\left(m_{\mathrm{r}}\right)_{\|}=\frac{M_{1}+M_{3}}{2}-\frac{\left(M_{1}-M_{3}\right)}{2} \cos 2 \theta
$$

$$
\left(m_{\mathrm{r}}\right)_{\perp}=\frac{M_{1}-M_{3}}{2} \sin 2 \theta
$$

Les coefficients des termes en $\sin 2 \theta$ et $\cos 2 \theta$ sont égaux et de signe contraire.

Pour que la relation tensorielle soit vérifiée il faut que les développements en série de Fourier de $\left(m_{\mathrm{r}}\right)$ soient de la forme :

$$
\begin{aligned}
& \left(m_{\mathrm{r}}\right)_{\|}=a_{0}+a_{2} \cos 2 \theta+\text { termes négligeables } \\
& \left(m_{\mathrm{r}}\right)_{\perp}=b_{2} \sin 2 \theta+\text { termes négligeables }
\end{aligned}
$$

et que l'on ait de plus $\left(a_{2}\right)_{\|}=-\left(b_{2}\right)_{\perp}$.

Le tableau $\mathrm{V}$ donne les résultats obtenus pour les quatre grandeurs dans le cas où $k^{\prime}=\lambda_{3}=1,5$. Seule l'ATR peut être représentée par un tenseur de rang $2\left({ }^{3}\right)$. Pour les autres grandeurs on a bien la bonne forme de développement en série mais la condition $\left(a_{2}\right)_{\|}=-\left(b_{2}\right)_{\perp}$ n'est pas vérifiée. Nous remarquons également (Tab. V) que les composantes parallèles varient peu entre grandeurs et grandeurs déformées, contrairement aux composantes perpendiculaires qui sont l'image du déphasage. Cette remarque est également illustrée pour l'ATR par les figures IIIa, $a^{\prime}$ et IIIb, $b^{\prime}$.

\subsubsection{Susceptibilité relative. Validité de l'approxima-} tion tensorielle: linéarité du milieu. Développement en série de Fourier des indicatrices. - Rappelons les résultats du paragraphe 4.1. Dans le cas d'un milieu linéaire la susceptibilité est représentée par un tenseur de rang 2 qui rapporté à ses axes principaux,

( $\left.{ }^{3}\right)$ Remarque : Les termes du développement en série de Fourier d'ordre supérieur à 2 ne sont pas significatifs pour les raisons invoquées plus haut. Les valeurs des $M_{\mathrm{TR}}$ déterminées par cette méthode sont les mêmes que celles déduites de l'assimilation à la transformation linéaire précédente, aux imprécisions de calcul numérique près.

Tableau II. - Analyse fine de la forme des indicatrices; développement en série de Fourier. Similitude des fabriques mécanique et magnétique. Caractère linéaire de l'ATR.

[Detailed analysis of the shape of indicatrices ; Fourier series expansion ; similarity of the mechanical and magnetic fabrics ; linearity of the TRM.]

\begin{tabular}{|c|c|c|c|c|c|c|c|c|c|}
\hline \multicolumn{1}{|c|}{ ATR } & \multicolumn{1}{|c|}{} \\
\hline$\lambda_{3}$ & $a_{0}$ & $a_{2}$ & $10^{4} a_{4}$ & $10^{4} a_{6}$ & $10^{4} a_{8}$ & $\operatorname{tg} \theta_{0} / \operatorname{tg} \theta_{1}$ & $\left\{\left(a_{0}-a_{2}\right) /\left(a_{0}+a_{2}\right)\right\}^{1 / 2}$ & $M_{3} / M_{1}$ & $M_{3} M_{1}^{2}$ \\
\hline 0,6666 & 1,246 & 0,4427 & -6 & -5 & -3 & 0,6896 & 0,6898 & 0,6899 & 0,9572 \\
\hline 0,5 & 1,636 & 0,9258 & -33 & -24 & -14 & 0,5254 & 0,5265 & 0,5254 & 0,8796 \\
\hline 1,5 & 0,9731 & $-0,3348$ & -5 & +2 & -3 & 1,431 & 1,431 & 1,432 & 0,9570 \\
\hline 2 & 1,058 & $-0,5722$ & -17 & -14 & -9 & 1,834 & 1,830 & 1,834 & 0,8790 \\
\hline
\end{tabular}


Tableau III. - Analyse fine de la forme des indicatrices; développement en série de Fourier. Similitude des fabriques mécanique et magnétique. Caractère non linéaire des autres grandeurs: cas de l'ARIS. $\left(\theta_{1}\right)_{1}$ : valeur de $\theta$, calculée dans l'approximation linéaire définie par a et $a_{2} .\left(M_{\mathrm{r}}\right)_{1}$ : valeur de l'ARIS calculée dans la même approximation.

[Detailed analysis of the shape of indicatrices; Fourier series expansion ; similarity of the mechanical and magnetic fabrics; lack of linearity of the other magnetic quantities; case of the SIRM. $\left(\theta_{1}\right)_{1}$ : value of $\theta$ calculated in the linear approximation defined by $a$ and $a_{2}\left(M_{\mathrm{r}}\right)_{1}$ value of the SIRM calculated in the same approximation.]

\begin{tabular}{|c|c|c|c|c|}
\hline$\theta_{0}$ & $\theta_{1}$ & $\left(\theta_{1}\right)_{1}$ & $\left(M_{\mathrm{r}}\right)_{1}$ cal & $M_{\mathrm{r}}$ \\
\hline 0 & 0 & 0 & 1,332 & 1,331 \\
\hline 10 & 3,809 & 6,098 & 1,319 & 1,326 \\
\hline 20 & 7,804 & 12,43 & 1,282 & 1,311 \\
\hline 30 & 12,21 & 19,28 & 1,222 & 1,284 \\
\hline 40 & 17,34 & 26,95 & 1,145 & 1,242 \\
\hline 50 & 23,71 & 35,83 & 1,056 & 1,180 \\
\hline 60 & 32,26 & 46,83 & 0,797 & 1,093 \\
\hline 70 & 44,69 & 59,60 & 0,882 & 0,9789 \\
\hline 80 & 63,68 & 72,77 & 0,764 & 0,8607 \\
\hline 90 & 90 & 90 & 0,807 & 0,8044 \\
\hline
\end{tabular}

$\left(\theta_{1}\right)_{1}$ : Valeur de $\theta$, calculée dans l'approximation linéaire définie par $a_{0}$ et $a_{2}$.

$\left(M_{\mathrm{r}}\right)_{1}$ : Valeur de l'ARIS calculée dans la même approximation.
Tableau V. - Aimantation rémanente parallèle et perpendiculaire au champ magnétisant: caractère tensoriel de l'aimantation rémanente. Ces valeurs correspondent à $k^{\prime}=\lambda_{3}=1,5$.

[Remanent magnetization measured parallel and perpendicular to the magnetizing field: tensorial character of the remanent magnetization. The figures is this table correspond to $k^{\prime}=\lambda_{3}=1.5$.]

\begin{tabular}{|l|c|c|c|}
\cline { 2 - 4 } \multicolumn{1}{c|}{} & \multicolumn{2}{c|}{$\left(M_{\mathrm{r}}\right)_{\|}$} & $\left(m_{\mathrm{r}}\right)_{\perp}$ \\
\cline { 2 - 4 } \multicolumn{1}{c|}{} & $a_{0}$ & $a_{2}$ & $b_{2}$ \\
\hline ARIS & 1,038 & 0,1159 & $-0,2318$ \\
\hline ARIS $D$ & 1,032 & 0,1178 & $-0,3801$ \\
\hline ATR & 1,062 & 0,1886 & $-0,1887$ \\
\hline ATR $D$ & 1,065 & 0,1823 & $-0,3565$ \\
\hline
\end{tabular}

Ces valeurs correspondent à $k^{\prime}=\lambda_{3}=1,5$.

qui sont ici les axes principaux de déformations, s'écrit :

$$
\left[\begin{array}{lll}
S_{1} & & \\
& S_{2} & \\
& & S_{3}
\end{array}\right]=\left[\begin{array}{lll}
\left(a_{0}-a_{2}\right) & & \\
& \left(a_{0}-a_{2}\right) & \\
& & \left(a_{0}+a_{2}\right)
\end{array}\right] .
$$

Le développement en série de Fourier de l'indicatrice ne comporte qu'un terme constant et un terme en $\cos 2 \theta$. Pour qu'on puisse faire l'approximation linéaire il faut que les termes d'ordre supérieur à 2 du développement soient négligeables.

Le tableau VI montre la validité et les limites de l'approximation linéaire en fonction de $\lambda_{3}$ pour diverses valeurs de $R$. Elle est d'autant plus justifiée que $R$ est petit et que la déformation est faible. Elle est acceptable pour $\lambda_{3}$ compris entre 2 et 0,5 et

Tableau IV. - Analyse fine de la forme des indicatrices; développement en série de Fourier. Similitude des fabriques mécanique et magnétique. Caractère non linéaire des autres grandeurs : cas de l'ARIS.

[Detailed analysis of the shape of indicatrices ; Fourier series expansion ; similarity of the mechanical and magnetic fabrics ; lack of linearity of the other magnetic quantities : case of the SIRM.]

\begin{tabular}{|c|c|c|c|c|c|c|c|c|c|}
\hline \multicolumn{1}{|c|}{ ARIS } & \multicolumn{1}{l|}{$\mid$} \\
\hline$\lambda_{3}$ & $a_{0}$ & $a_{2}$ & $10^{4} a_{4}$ & $10^{4} a_{6}$ & $10^{4} a_{8}$ & $\operatorname{tg} \theta_{0} / \operatorname{tg} \theta_{1}$ & $\left(\left(a_{0}-a_{2}\right) /\left(a_{0}+a_{2}\right)\right\}^{1 / 2}$ & $M_{3} / M_{1}$ & $M_{3} M_{1}^{2}$ \\
\hline 2,5 & 1,048 & $-0,4854$ & -16 & +32 & +14 & 2,6972 & 1,6503 & 1,6546 & 0,8612 \\
\hline
\end{tabular}


Tableau VI. - Susceptibilité relative. Validité de l'approximation tensorielle : linéarité du milieu. Développement en série de Fourier des indicatrices.

[Relative susceptibility. Validity of the tensorial approximation : linearity of the medium. Fourier series expansion of the indicatrices.]

\begin{tabular}{|l|c|c|c|c|c|c|c|c|c|}
\hline \multicolumn{1}{|c|}{$\lambda_{3}$} & $a_{0}$ & $a_{2}$ & $a_{4}$ & $a_{6}$ & $a_{8}$ & $a_{\mathrm{m}}$ & $\left(a_{\mathrm{m}}\right)_{1}$ & $\Delta a_{\mathrm{m}} / a_{\mathrm{m}}$ & \\
\hline 10 & 1,507 & 1,363 & 0,6246 & 0,2858 & 0,1306 & 0,1422 & 0,0500 & 64,85 & \\
\hline 4 & 1,275 & 0,7701 & 0,2399 & 0,0748 & 0,0233 & 0,2874 & 0,2470 & 14,06 & \\
\hline 2 & 1,119 & 0,3469 & 0,0569 & 0,0093 & 0,0016 & 0,5334 & 0,5270 & 0,55 & \\
\hline 1,5 & 1,065 & 0,1918 & 0,0186 & 0,0020 & 0,0003 & 0,6967 & 0,6949 & 0,26 & $R=10$ \\
\hline 0,6666 & 0,9483 & $-0,1578$ & 0,0151 & $-0,0015$ & 0,0002 & 1,396 & 1,399 & 0,21 & $x=0,9801$ \\
\hline 0,5 & 0,9194 & $-0,2491$ & 0,0396 & $-0,0065$ & 0,0010 & 1,726 & 1,743 & 0,98 & \\
\hline 0,25 & 0,8713 & $-0,4070$ & 0,1184 & $-0,0357$ & 0,0109 & 2,607 & 2,752 & 5,57 & \\
\hline 0,1 & 0,8402 & $-0,5150$ & 0,2099 & $-0,0907$ & 0,0401 & 3,660 & 4,167 & 13,85 & \\
\hline
\end{tabular}

\begin{tabular}{|l|c|c|c|c|c|c|c|c|c|}
\hline$\lambda_{3}$ & $a_{0}$ & $a_{2}$ & $a_{4}$ & $a_{6}$ & $a_{8}$ & $a_{\mathrm{m}}$ & $\left(a_{\mathrm{m}}\right)_{1}$ & $\begin{array}{c}10^{2} \\
\Delta a_{\mathrm{m}} / a_{\mathrm{m}}\end{array}$ & \\
\hline 4 & 1,494 & 1,336 & 0,5920 & 0,2593 & 0,1129 & 0,1468 & 0,0559 & 61,88 & \\
\hline 2 & 1,196 & 0,5606 & 0,1322 & 0,0308 & 0,6072 & 0,3851 & 0,3619 & 6 & $R=50$ \\
\hline 0,5 & 0,8823 & $-0,3685$ & 0,0848 & $-0,0198$ & 0,0043 & 2,337 & 2,434 & 4,14 & $x=0,9992$ \\
\hline 0,25 & 0,8180 & $-0,5886$ & 0,2468 & $-0,1055$ & 0,0451 & 4,568 & 6,131 & 34,21 & \\
\hline
\end{tabular}

\begin{tabular}{|l|c|c|c|c|c|c|c|c|c|}
\hline$\lambda_{3}$ & $a_{0}$ & $a_{2}$ & $a_{4}$ & $a_{6}$ & $a_{8}$ & $a_{\mathrm{m}}$ & $\left(a_{\mathrm{m}}\right)_{1}$ & $\begin{array}{c}10^{2} \\
\Delta a_{\mathrm{m}} / a_{\mathrm{m}}\end{array}$ \\
\hline 4 & 1,680 & 1,802 & 0,9215 & 0,4596 & 0,2261 & 0,4918 & $-0,220$ & $?$ & $R=500$ \\
\hline 2 & 1,255 & 0,7220 & 0,1980 & 0,0527 & 0,0137 & 6,307 & 0,2698 & 12,11 & $x=0,9999$ \\
\hline 0,5 & 0,8574 & $-0,4539$ & 0,1209 & $-0,0302$ & 0,0074 & 2,950 & 3,249 & 10,14 & March \\
\hline 0,25 & 0,7819 & 0,7159 & 0,3514 & $-0,1712$ & 0,0833 & 7,226 & 22,613 & 214 & \\
\hline
\end{tabular}

correspond à une erreur relative sur les anisotropies qui est : meilleure que $10^{-2}$ pour $R=10$, de l'ordre de $5 \times 10^{-2}$ pour $R=50$ et de l'ordre de $10^{-1}$ pour $R=500$. Pour des marqueurs très allongés et pour de fortes déformations, les termes pairs d'ordre supérieur deviennent prépondérants et l'approximation n'a plus de sens.

\section{Conclusion.}

Ce travail montre qu'il est possible d'obtenir des relations quantitatives entre l'anisotropie magnétique et l'anisotropie structurale d'une roche dans le cas envisagé d'un mécanisme d'O.P.F. Une telle démarche a d'abord pour but la compréhension de la physique des phénomènes à partir de bases simples de manière à pouvoir mettre en évidence les paramètres du problème.

Le modèle d'O.P.F. développé par Fernandez nous semble solide. Il a été expérimenté par cet auteur, dans le cas d'un problème plan, tant pour 
des déformations coaxiales que pour des cisaillements simples. Fernandez a également montré que les interactions mécaniques entre marqueurs ne modifient pas de façon cruciale les résultats obtenus. Mais l'O.P.F. n'est pas le seul mécanisme de déformation à l'échelle du grain qui conduise à une orientation préférentielle, nous en citerons deux autres. La déformation interne du marqueur par activation de plans de glissement cristallographiques conduit à une rotation avec changement de forme et à une orientation préférentielle des axes cristallographiques. Dans ce cas et pour des roches monominérales on peut établir une relation entre fabrique structurale et déformation. L'autre est le phénomène de dissolution cristallisation. Le grain enrobé d'un milieu acqueux est soumis à un gradient de pression. Il y a dissolution de la matière dans la zone de forte pression et dépôt de celle-ci dans la partie abritée. Il en résulte une orientation préférentielle de forme, alors que les axes cristallographiques ne sont pas réorientés. Dans ce cas, d'une grande importance pratique, il n'est pas évident d'établir une relation quantitative entre fabrique structurale et déformation. Ces relations quantitatives traduisant l'orientation préférentielle sont le point de départ obligé d'un modèle d'anisotropie magnétique qui les caractérise. Les spécialistes de magnétisme sont largement tributaires des travaux des mécaniciens.

L'aspect magnétique du modèle privilégie les processus d'aimantation qui en sont la base physique. Ceux que nous avons retenus sont bien connus, quoique idéalisés pour les besoins du calcul.

Cette étude montre l'importance de la morphologie des constituants de la roche. L'état de division de la matière conditionne largement les mécanismes d'aimantation et le choix des grandeurs physiques à mesurer.

L'énergie liée au champ démagnétisant de forme joue un rôle prépondérant.

Dans un modèle cohérent plusieurs grandeurs magnétiques peuvent conduire à des résulats comparables dès lors que leur choix est représentatif des processus d'aimantation mis en jeu.

La nature de certains mécanismes d'aimantation élémentaires conduit en général à un milieu non linéaire doué de mémoire. Le fait d'envisager le cas plus général où il existe des interactions magnétiques entre porteurs ne peut qu'accentuer ce caractère. De ce fait les propriétés magnétiques de la roche dépendent à la fois de son histoire magnétique et tectonique. Cette situation doit être prise en compte dans le choix des grandeurs magnétiques et de leur protocole de mesure. Elle ne permet pas en général l'assimilation à un milieu linéaire dont l'anisotropie magnétique est caractérisée par un tenseur de rang deux. La similitude entre fabrique magnétique et fabrique structurale nécessite une réflexion sur la façon dont on peut associer un milieu linéaire au milieu réel que constitue la roche. L'un de nous a précisé ce point de vue à propos de l'anisotropie de susceptibilité magnétique [14].

Dans une roche polyminérale tous les constituants sont «magnétiques » et l'information globale est généralement complexe. Son analyse fine par un ensemble de techniques appropriées où les paramètres champ magnétique et température sont fondamentaux permet, dans des cas favorables, de montrer le rôle prépondérant d'un, ou d'un petit nombre, de constituants. Il devient alors possible de développer des modèles qui les prennent en compte de façon spécifique. C'est là une deuxième étape où la minéralogie magnétique joue un rôle de premier plan. Il faut cependant préciser que la seule connaissance de l'information magnétique ne suffit pas en général pour résoudre complètement le problème et doit être complétée par les techniques classiques ou élaborées utilisées habituellement en analyse structurale.

\section{Remerciements.}

L'un de nous a rédigé cet article pour des raisons évidentes d'homogénéité. Il n'aurait pu le faire sans une étroite collaboration avec ses collègues coauteurs.

Ce dernier remercie G. Aubert pour l'intérêt qu'il a porté à ce travail ; ces remarques et suggestions l'ayant largement fait évoluer.

Les discussions qu'il a eues avec A. Fernandez et J. L. Porteseil lui ont permis de préciser certains aspects du modèle.

L'aide de M. Jerjini et P. Molho dans le domaine de l'informatique lui ont grandement facilité l'acquisition et la présentation des résultats numériques.

\section{Annexe I.}

Calcul de la susceptibilité en champ faible d'un ensemble de marqueurs idéalement polydomaines.

Soit (R) le repère $X, Y, Z$ dans lequel on a défini la densité $D(\theta, \phi)$ et soit $\left(\mathrm{R}^{\prime}\right)$ un repère $X^{\prime}$, $Y^{\prime}, Z^{\prime}$ lié au grain dont l'axe est suivant $\pm \mathbf{u}(\theta, \phi)$ (cf. Fig. A.1)

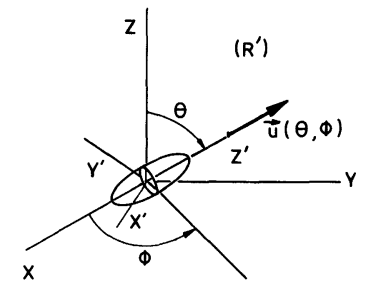

$\left(\mathrm{R}^{\prime}\right)$ est défini comme ayant l'axe $Z^{\prime}$ suivant $\mathbf{u}$. Les vecteurs unitaires de $\left(R^{\prime}\right): \mathbf{i}^{\prime}, \mathbf{j}^{\prime}, \mathbf{k}^{\prime}$, ont pour 
composantes $\mathbf{i}^{\prime}\left(l_{1}^{\prime}, m_{1}^{\prime}, n_{1}^{\prime}\right) ; \mathbf{j}^{\prime}\left(l_{2}^{\prime}, m_{2}^{\prime}, n_{2}^{\prime}\right)$; $\mathbf{k}^{\prime}\left(l_{3}^{\prime}, m_{3}^{\prime}, n_{3}^{\prime}\right)$. On passe de $\left(\mathrm{R}^{\prime}\right)$ à $(\mathrm{R})$ par les relations :

$$
\left[\begin{array}{l}
X^{\prime} \\
Y^{\prime} \\
Z^{\prime}
\end{array}\right]=\left[\begin{array}{lll}
l_{1}^{\prime} & m_{1}^{\prime} & n_{1}^{\prime} \\
l_{2}^{\prime} & m_{2}^{\prime} & n_{2}^{\prime} \\
l_{3}^{\prime} & m_{3}^{\prime} & n_{3}^{\prime}
\end{array}\right]\left[\begin{array}{c}
X \\
Y \\
Z
\end{array}\right]
$$

h étant le vecteur unitaire du champ appliqué $\mathbf{H}$ repéré par rapport à $(\mathrm{R}): \mathbf{h}\left(X_{0}, Y_{0}, Z_{0}\right)$ son expression dans les axes liés au grain sera: $\mathbf{h}^{\prime}$ $\left(X_{0}^{\prime}, Y_{0}^{\prime}, Z_{0}^{\prime}\right)$, les grandeurs primées étant liées aux grandeurs non primées par la relation de passage de $\left(R^{\prime}\right)$ à $(R)$.

Le grain considéré voit le champ de direction $\mathbf{h}^{\prime}$. La nullité du champ interne implique que l'aimantation soit alignée avec $\mathbf{h}$ et que l'on ait $\mathbf{H}^{\prime}=N^{\prime} \mathbf{M}^{\prime}$, soit : $\frac{M^{\prime}}{H^{\prime}}=N^{\prime} ; N^{\prime}$ étant le coefficient de champ démagnétisant dans la direction $h^{\prime}$. $N^{\prime}$ exprime dans ce cas particulier en fonction des coefficients de champ démagnétisant $N_{1}, N_{2}, N_{3}$ du grain et de la direction $\mathbf{h}^{\prime}$ par la relation :

$$
N_{\mathbf{h}^{\prime}}^{\prime}=N_{1} X_{0}^{\prime 2}+N_{2} Y_{0}^{\prime 2}+N_{3} Z_{0}^{\prime 2} .
$$

L'ellipsoïde étant de révolution on a $N_{1}=N_{2}$ et comme $N_{1}+N_{2}+N_{3}=1$ il vient $N_{1}=N_{2}=$ $\frac{1-N_{3}}{2}$. De plus $X_{0}^{\prime 2}+Y_{0}^{\prime 2}+Z_{0}^{\prime 2}=1$, soit pour $N_{\mathbf{h}^{\prime}}^{\prime}$

$$
N_{\mathbf{h}^{\prime}}^{\prime}=\frac{\left(1-N_{3}\right)+\left(3 N_{3}-1\right) Z_{0}^{\prime 2}}{2}
$$

La susceptibilité $s$ du grain soumis au champ de direction $\mathbf{h}$ a pour expression :

$$
s=\frac{1}{N_{\mathbf{h}^{\prime}}^{\prime}}=\frac{2}{\left(1-N_{3}\right)+\left(3 N_{3}-1\right) Z_{0}^{\prime 2}} .
$$

Soit en exprimant $Z_{0}^{\prime 2}$ dans (R) :

$$
\begin{aligned}
s & =\frac{1}{N_{\mathbf{h}}} \\
& =\frac{2}{\left(1-N_{3}\right)+\left(3 N_{3}-1\right)\left(X X_{0}+Y Y_{0}+Z Z_{0}\right)^{2}}
\end{aligned}
$$

On a $\mathrm{d} N$ grains dont l'axe pointe dans la direction $\pm \mathbf{u}$ et la susceptibilité de l'ensemble des grains dans la direction h s'écrit :

$$
\begin{aligned}
\left(s_{\mathrm{c}}\right)_{\mathbf{h}} & =\int_{0}^{\pi / 2} \int_{0}^{2 \pi} \times \\
& \times \frac{2}{\left(1-N_{3}\right)+\left(3 N_{3}-1\right)(\mathbf{u} \cdot \mathbf{h})^{2}} D_{0} D(\theta, \phi) \mathrm{d} S .
\end{aligned}
$$

Pour une répartition isotrope des grains $D_{0}=$ $\frac{N}{2 \pi}$ et $D=1$.

REVUE DE PHYSIQUE APPLIQUÉE. - T. 23, N 5, MAI 1988
On a :

$$
\begin{aligned}
\left(s_{\mathrm{e}_{0}}\right)_{\mathbf{h}}=2 D_{0} \int_{0}^{\pi / 2} \int_{0}^{2 \pi} \times & \frac{1}{\left(1-N_{3}\right)+\left(3 N_{3}-1\right)(\mathbf{u} \cdot \mathbf{h})^{2}} \mathrm{~d} S .
\end{aligned}
$$

La susceptibilité relative traduisant l'O.P.F. s'écrit :

$$
\begin{aligned}
S_{\mathrm{h}}=\frac{\left(s_{\mathrm{c}}\right)_{\mathrm{h}}}{\left(s_{\mathrm{e}_{0}}\right)_{\mathrm{h}}}=\frac{2 \pi \int_{0}^{\pi / 2} \int_{0}^{2 \pi} F(\theta, \phi) D(\theta, \phi) \mathrm{d} S}{} & \left\{\int_{0}^{\pi / 2} \int_{0}^{2 \pi} F(\theta, \phi) \mathrm{d} S\right\} \times \\
& \times\left\{\int_{0}^{\pi / 2} \int_{0}^{2 \pi} D(\theta, \phi) \mathrm{d} S\right\}
\end{aligned}
$$

avec $: F(\theta, \phi)=\left\{1-N_{3}+\left(3 N_{3}-1\right)(\mathbf{u} \cdot \mathbf{h})^{2}\right\}^{-1}$.

Cette expression permet d'établir l'indicatrice de la susceptibilité relative par rapport aux axes principaux de déformation.

Valeur du coefficient de champ démagnétisant $N_{3}$ pour des ellipsoïdes allongés.

$N_{3}$ s'exprime en fonction du rapport $m$ du demiaxe de révolution au rayon de la section circulaire par :

$$
N_{3}=\frac{1}{m^{2}-1}\left[\frac{m}{\sqrt{m^{2}-1}} \log \left(m+\sqrt{m^{2}-1}\right)-1\right]
$$

pour $1<m<\infty$. On a de plus :

$$
\begin{array}{cl}
m_{\infty} \rightarrow N_{3}=0 & \text { marqueur linéaire } \\
m=1 \rightarrow N_{3}=1 / 3 & \text { marqueur sphérique . }
\end{array}
$$

La relation précédente est valable pour les valeurs intermédiaires de $m$.

Le paramètre $\chi$ du grain vaut : $\frac{m^{2}-1}{m^{2}+1}$; soit $m=\left(\frac{1+\chi}{1-\chi}\right)^{1 / 2}$.

$N_{3}$ s'exprime en fonction de $\chi$ par la relation :

$$
\begin{aligned}
N_{3}=\frac{1-\chi}{1+2 \chi}\left[\left(\frac{1+\chi}{2 \chi}\right)^{1 / 2} \times\right. \\
\\
\left.\quad \times \log \left(\frac{\sqrt{1+\chi}+\sqrt{2 \chi}}{\sqrt{1-\chi}}\right)-1\right]
\end{aligned}
$$

pour $0<\chi<1$.

On a de plus :

$$
\begin{array}{ll}
\chi=1 \rightarrow N_{3}=0 & \text { marqueurs linéaires } \\
\chi=0 \rightarrow N_{3}=1 / 3 & \text { marqueurs sphériques . }
\end{array}
$$

La relation précédente est valable pour les valeurs intermédiaires de $\chi$. 


\section{Annexe II.}

Le matériau qui constitue le marqueur suit une loi de Rayleigh.

Calcul de l'anisotropie de diverses grandeurs magnétiques résultant d'une O.P.F. des grains : susceptibilité réversible et irréversible, aimantation, aimantation rémanente.

Le matériau constituant le grain est isotrope et son aimantation est de la forme $M=a h+b h^{2}, h$ est le champ interne c'est-à-dire le champ appliqué corrigé du champ démagnétisant de forme.

Pour la suite de ce calcul nous aurons besoin de deux repères (cf. Fig. A.2). L'un $\mathrm{R}(X, Y, Z)$, est fixe, l'autre $\mathrm{R}^{\prime}\left(X, Y^{\prime}, Z^{\prime}\right)$ est lié aux grains dont l'axe de révolution est suivant $\pm \mathbf{u}(\theta, \phi) . D(\theta, \phi)$ est défini dans $(R)$.

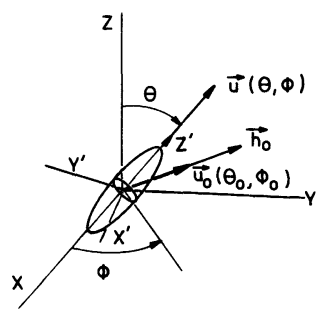

Les relations de passage s'écrivent :

$$
\begin{aligned}
& {\left[\begin{array}{c}
X^{\prime} \\
Y^{\prime} \\
Z^{\prime}
\end{array}\right]=\left[\begin{array}{lll}
l_{1}^{\prime} & m_{1}^{\prime} & n_{1}^{\prime} \\
l_{2}^{\prime} & m_{2}^{\prime} & n_{2}^{\prime} \\
l_{3}^{\prime} & m_{3}^{\prime} & n_{3}^{\prime}
\end{array}\right]\left[\begin{array}{c}
X \\
Y \\
Z
\end{array}\right] ;} \\
& {\left[\begin{array}{c}
X \\
Y \\
Z
\end{array}\right]=\left[\begin{array}{ccc}
l_{1}^{\prime} & l_{2}^{\prime} & l_{3}^{\prime} \\
m_{1}^{\prime} & m_{2}^{\prime} & m_{3}^{\prime} \\
n_{1}^{\prime} & n_{2}^{\prime} & n_{3}^{\prime}
\end{array}\right]\left[\begin{array}{c}
X^{\prime} \\
Y^{\prime} \\
Z^{\prime}
\end{array}\right] .}
\end{aligned}
$$

Les vecteurs unitaires $\mathbf{i}^{\prime}, \mathbf{j}^{\prime}, \mathbf{k}^{\prime}$ de $\left(R^{\prime}\right)$ ont pour composantes :

$$
\begin{aligned}
& \mathbf{i}^{\prime}\left|\begin{array}{l|r}
l_{1}^{\prime} & =\sin \phi \\
m_{1}^{\prime} & =-\cos \phi ; \quad \mathbf{j}^{\prime} \\
n_{1}^{\prime} & =0
\end{array}\right| \begin{aligned}
l_{2}^{\prime} & =\cos \theta \cos \phi \\
m_{2}^{\prime} & =\cos \theta \sin \phi ; \\
n_{2}^{\prime} & =-\sin \theta
\end{aligned} ; \\
& \mathbf{k}^{\prime} \mid \begin{array}{c}
l_{3}^{\prime}=\sin \theta \cos \phi \\
m_{3}^{\prime}=\sin \theta \sin \phi \\
n_{3}^{\prime}=\cos \theta \\
\mathbf{k}^{\prime} \equiv \mathbf{u}
\end{array}
\end{aligned}
$$

Appliquons un champ magnétique $\mathbf{h}_{0}=\mathbf{u}_{0} h_{0}$ de direction $\left(\theta_{0}, \phi_{0}\right)$. Dans $\left(\mathrm{R}^{\prime}\right)$ on a $\mathrm{u}_{0}^{\prime} h_{0}$. L'aimantation résultant de ce champ s'écrit dans $\left(R^{\prime}\right)$ :

$$
\begin{aligned}
& M_{1}^{\prime}=a_{1}^{\prime} X_{0}^{\prime} h_{0}+b_{1}^{\prime} X_{0}^{\prime 2} h_{0}^{2} \\
& M_{2}^{\prime}=a_{2}^{\prime} Y_{0}^{\prime} h_{0}+b_{2}^{\prime} Y_{0}^{\prime 2} h_{0}^{2} \\
& M_{3}^{\prime}=a_{3}^{\prime} Z_{0} h_{0}+b_{3}^{\prime} Z_{0}^{2} h_{0}^{2} .
\end{aligned}
$$

En effet, suivant les axes l'aimantation est alignée avec le champ interne; ce dernier vaut: $h_{i}=$
$h_{0}-N_{i} M_{i}$. En tenant compte du fait que le matériau suit une loi de Rayleigh et en se bornant aux champs faibles on peut faire un développement en série des $M_{i}$. En ne gardant que les termes jusqu'à l'ordre 2 en $h_{0}$ on obtient les expressions des $M_{i}$. On a une loi Rayleigh suivant les axes et les nouveaux coefficients valent :

$$
\begin{aligned}
a_{i}^{\prime}= & \frac{a}{1+a N_{i}} \\
b_{i}^{\prime}= & \frac{b}{1+a N_{i}} \times \\
& \times\left(1-\frac{2 a N_{i}}{\left(1+a N_{i}\right)^{2}}+\frac{a^{2} N_{i}}{\left(1+a N_{i}\right)^{3}}\right)
\end{aligned}
$$

où les $N_{i}$ sont les coefficients de champ démagnétisant de l'ellipsoïde.

$$
N_{1}=N_{2}=\frac{1-N_{3}}{2} .
$$

L'aimantation suivant la direction du champ magnétisant s'écrit :

$$
\begin{aligned}
\left(M^{\prime}\right)_{\mathrm{h}_{0}}= & M_{1}^{\prime} X_{0}^{\prime}+M_{2}^{\prime} Y_{0}^{\prime}+M_{3}^{\prime} Z_{0}^{\prime} \\
= & h_{0}\left\{a_{1}^{\prime} X_{0}^{\prime 2}+a_{2}^{\prime} Y_{0}^{\prime 2}+a_{3}^{\prime} Z_{0}^{\prime 2}\right\} \\
& +h_{0}^{2}\left\{b_{1}^{\prime} X_{0}^{\prime 3}+b_{2}^{\prime} Y_{0}^{\prime 3}+b_{3}^{\prime} Z_{0}^{\prime 3}\right\}
\end{aligned}
$$

soit pour la susceptibilité :

$$
\frac{\partial\left(M^{\prime}\right) \mathbf{h}_{0}}{\partial h_{0}}=\left\{a_{1}^{\prime} X_{0}^{\prime 2}+a_{2}^{\prime} Y_{0}^{\prime 2}+a_{3}^{\prime} Z_{0}^{\prime 2}\right\} .
$$

Le premier terme correspond à la susceptibilité réversible, le second à la susceptibilité irréversible.

\section{Susceptibilité réversible relative $\left(S_{\mathrm{r}}\right)_{\mathrm{h}_{0}}$}

La susceptibilité réversible dans la direction du champ $\mathbf{h}_{0}$ s'écrit :

$$
\begin{aligned}
\left(s_{\mathrm{r}}\right)_{\mathrm{h}_{0}}=\int_{0}^{\pi / 2} \int_{0}^{2 \pi}\left\{a_{1}^{\prime}+\left(a_{3}^{\prime}-a_{1}^{\prime}\right)\right. & \left.Z_{0}^{2}\right\} \times \\
& \times D_{0} D(\theta, \phi) \mathrm{d} S
\end{aligned}
$$

soit en repassant dans $(R)$ :

$$
\begin{aligned}
\left(s_{\mathrm{r}}\right)_{\mathrm{h}_{0}}=\int_{0}^{\pi / 2} \int_{0}^{2 \pi}\left\{a_{1}^{\prime}+\left(a_{3}^{\prime}-a_{1}^{\prime}\right)\left(\mathbf{u} \cdot \mathbf{u}_{0}\right)^{2}\right\} \times \\
\times D_{0} D(\theta, \phi) \mathrm{d} S .
\end{aligned}
$$

Pour un ensemble de marqueurs orientés au hasard $\left(s_{\mathrm{r}}\right)_{0}$ s'obtient en faisant $D_{0}=\frac{N}{2 \pi}$ et $D=1 ;$ on a :

$$
\left(s_{\mathrm{r}}\right)_{0}=N \frac{2 a_{1}^{\prime}+a_{3}^{\prime}}{3} .
$$


Il en résulte pour la susceptibilité relative :

$$
\begin{aligned}
\left(S_{\mathrm{r}}\right)_{\mathrm{h}_{0}}=\frac{\left(s_{\mathrm{r}}\right)_{\mathrm{h}_{0}}}{\left(s_{\mathrm{r}}\right)_{0}}= & \frac{3}{2 a_{1}^{\prime}+a_{3}^{\prime}} \times \\
& \times \frac{\int_{0}^{\pi / 2} \int_{0}^{2 \pi} F(\theta, \phi) D(\theta, \phi) \mathrm{d} S}{\int_{0}^{\pi / 2} \int_{0}^{2 \pi} D(\theta, \phi) \mathrm{d} S}
\end{aligned}
$$

avec : $F(\theta, \phi)=\left\{a_{1}^{\prime}+\left(a_{3}^{\prime}-a_{1}^{\prime}\right)\left(\mathbf{u} \cdot \mathbf{u}_{0}\right)^{2}\right\}$.

Susceptibilité irréversible relative $\left(S_{\mathrm{ir}}\right)_{\mathrm{h}_{0}}$

Le calcul est en tout point analogue. Posons :

$$
\left(\left(s_{1}\right)_{\mathrm{ir}}\right)_{\mathrm{h}_{0}}=\left\{b_{1}^{\prime} X_{0}^{\prime 3}+b_{2}^{\prime} Y_{0}^{\prime 3}+b_{3}^{\prime} Z_{0}^{\prime 3}\right\}
$$

soit dans (R) :

$$
\begin{aligned}
\left(\left(s_{1}\right)_{\mathrm{ir}}\right)_{\mathrm{h}_{0}}= & b_{1}^{\prime}\left\{l_{1}^{\prime} X_{0}+m_{1}^{\prime} Y_{0}+n_{1}^{\prime} Z_{0}\right\}^{3} \\
& +b_{2}^{\prime}\left\{l_{2}^{\prime} X_{0}+m_{2}^{\prime} Y_{0}+n_{2}^{\prime} Z_{0}\right\}^{3} \\
& +b_{3}^{\prime}\left\{l_{3}^{\prime} X_{0}+m_{3}^{\prime} Y_{0}+n_{3}^{\prime} Z_{0}\right\}^{3}
\end{aligned}
$$

Pour l'ensemble des grains $\left(S_{\mathrm{ir}}\right)_{\mathrm{h}_{0}}$ s'écrit :

$$
\left(S_{\mathrm{ir}}\right)_{\mathrm{h}_{0}}=\int_{0}^{\pi / 2} \int_{0}^{2 \pi}\left(\left(s_{1}\right)_{\text {ir }}\right)_{\mathrm{h}_{0}} D_{0}(\theta, \phi) \mathrm{d} S .
$$

Pour une répartition au hasard des grains $\left(S_{\mathrm{i}_{0}}\right)_{\mathrm{h}_{0}}$ a pour valeur :

$$
\begin{aligned}
\left(S_{\mathrm{ir}_{0}}\right)_{\mathrm{h}_{0}}= & N\left\{\frac{b_{3}^{\prime}}{8} Z_{0}\left(3-Z_{0}^{2}\right)-\right. \\
& \left.-\frac{3 \pi}{32} b_{2}^{\prime} Z_{0}\left(1+Z_{0}^{2}\right)\right\} \\
= & N L .
\end{aligned}
$$

Soit pour $\left(S_{\mathrm{ir}}\right)_{\mathrm{h}_{0}}$ :

$$
\left(S_{\mathrm{ir}}\right)_{\mathrm{h}_{0}}=\frac{1}{L} \frac{\int_{0}^{\pi / 2} \int_{0}^{2 \pi}\left(\left(s_{1}\right)_{\mathrm{ir}}\right)_{\mathrm{h}_{0}} D(\theta, \phi) \mathrm{d} S}{\int_{0}^{\pi / 2} \int_{0}^{2 \pi} D(\theta, \phi) \mathrm{d} S} .
$$

\section{Aimantation}

Les composantes de l'aimantation d'un grain exprimées dans le repère $(R)$ valent :

$$
\begin{aligned}
\left(M_{1}\right)_{\mathrm{h}_{0}}=h_{0}\left\{l_{1}^{\prime} a_{1}^{\prime} X_{0}^{\prime}+l_{2}^{\prime} a_{2}^{\prime} Y_{0}^{\prime}+l^{\prime} a_{3}^{\prime} Z_{0}^{\prime}\right\}+ \\
+h_{0}^{2}\left\{l_{1}^{\prime} b_{1}^{\prime} X_{0}^{\prime 2}+l_{2}^{\prime} b_{2}^{\prime} Y_{0}^{\prime 2}+l_{3}^{\prime} b_{3}^{\prime} Z_{0}^{\prime 2}\right\}
\end{aligned}
$$

$\left(M_{2}\right)_{\mathbf{h}_{0}}$ et $\left(M_{3}\right)_{\mathbf{h}_{0}}$ ont des expressions analogues obtenues par permutation circulaire sur les $l^{\prime}$, $m^{\prime}, n^{\prime}$.

$X_{0}^{\prime}, Y_{0}^{\prime}, Z_{0}^{\prime}$ ont pour expression dans $(\mathrm{R})$ :

$$
\begin{aligned}
X_{0}^{\prime} & =l_{1}^{\prime} X_{0}+m_{1}^{\prime} Y_{0}+n_{1}^{\prime} Z_{0} \\
Y_{0}^{\prime} & =l_{2}^{\prime} X_{0}+m_{2}^{\prime} Y_{0}+n_{2}^{\prime} Z_{0} \\
Z_{0}^{\prime} & =l_{3}^{\prime} X_{0}+m_{3}^{\prime} Y_{0}+n_{3}^{\prime} Z_{0} .
\end{aligned}
$$

L'aimantation de l'ensemble des $N$ grains, compte tenu de l'O.P.F. a pour composantes :

$$
\left(M_{1 \mathrm{c}}\right)_{\mathrm{h}_{0}}=\int_{0}^{\pi / 2} \int_{0}^{2 \pi}\left(M_{1}\right)_{\mathrm{h}_{0}} D_{0} D(\theta, \phi) \mathrm{d} S
$$

et les quantités équivalentes pour $\left(M_{2 \mathrm{e}}\right)_{\mathrm{h}_{0}}$ et $\left(M_{3 \mathrm{c}}\right)_{\mathrm{h}_{0}}$.

Dans le cas où les axes des grains sont uniformément répartis, l'aimantation $\left(M_{1 \mathrm{e}}\right)$ de l'ensemble soumis au même champ $\mathbf{h}_{0}$ s'obtient en faisant $D_{0}=\frac{N}{2 \pi}$ et $D(\theta, \phi)=1$ dans l'expression de $\left(M_{1 \mathrm{c}}\right)_{\mathbf{h}_{0}}$.

L'aimantation relative $\left(m_{1}\right)_{\mathbf{h}_{0}}=\frac{\left(M_{1 \mathrm{e}}\right)_{\mathbf{h}_{0}}}{M_{10}}$ vaut

$$
\left(m_{1}\right)_{\mathbf{h}_{0}}=\frac{\int_{0}^{\pi / 2} \int_{0}^{2 \pi}\left(M_{1}\right)_{\mathrm{h}_{0}} D(\theta, \phi) \mathrm{d} S}{\int_{0}^{\pi / 2} \int_{0}^{2 \pi}\left(M_{1}\right)_{\mathrm{h}_{0}} \mathrm{~d} S}
$$

On a des expressions analogues pour $\left(M_{2}\right)_{\mathbf{h}_{0}}$ et $\left(M_{3}\right)_{\mathrm{h}_{0}}$. Le module de $(\mathbf{m})_{\mathrm{h}_{0}}$ et les angles $\theta$ et $\phi$ correspondants s'obtiennent en repassant en coordonnées sphériques. L'angle $\alpha=\left(\mathbf{m}, \mathbf{h}_{0}\right)$ que fait l'aimantation avec le champ inducteur a pour valeur :

$$
\alpha=\operatorname{Arccos} \frac{\mathbf{m} \cdot \mathbf{u}_{0}}{|\mathbf{m}|} .
$$

Dans le plan défini par $\mathbf{m}$ et $\mathbf{h}_{0}$, les composants de l'aimantation suivant le champ inducteur et perpendiculairement s'écrivent :

$$
\begin{aligned}
m_{\|} & =|\mathbf{m}| \cos \alpha \\
m_{\perp} & =|\mathbf{m}| \sin \alpha .
\end{aligned}
$$

Aimantation rémanente dans le domaine de Rayleigh

L'aimantation rémanente vaut : $M_{\mathrm{r}}=\frac{b}{2} h_{\mathrm{m}}^{2}, h_{\mathrm{m}}$ étant le champ maximum atteint. L'aimantation rémanente relative $\left(m_{\mathrm{r}}\right)_{\mathrm{h}_{0}}=\frac{\left(M_{\mathrm{r}}\right)_{\mathrm{h}_{0}}}{\left(M_{\mathrm{r}}\right)_{0}}$ est égale à $\left(S_{\text {ir }}\right)_{\mathbf{h}_{0}}$. 
Bibliographie générale

ASPECT MÉCANIQUE DU MODÈLE.

Jefferey, G. B., Proc. R. Soc. London, Ser. A 102 (1922) 161-179.

MARCH, Z. Kristallogr. 81 (1932) 285-297.

GAY, N. C., Tectonophys. 3 (1966) 539-564.

GAY, N. C., Tectonophys. 5 (1968) 211-234.

GAY, N. C., Tectonophys. 5 (1968) 295-302.

Tullis, T. E., PH. D. Thesis, Univ. Calif. Los Angeles (1971).

Tullis, T. E., Geol. Soc. Am. Bull. 87 (1976) 745-753.

OWENS, W. H., Tectonophys. 16 (1973) 249-261.

ReEd, L. J. et Tryggvason, E., Tectonophys. 24 (1974) 85-98.

Debat, P., Sirieys, P., Deramond, J. et Soula, J. C., Tectonophys. 28 (1975) 159-183.

Willis, D. G., Geol. Soc. Am. Bull. 88 (1977) 833-894.

Blanchard, J. Ph., Boyer, P. et GagnY, Cl., Tectonophys. 53 (1979) 1-25.

Fernandez, A., Thèse de Doctorat d'Etat, Université de Clermont Fd II (1984).

\section{AsPeCt MAGNÉTIQUe.}

Ouvrages généraux :

NÉEL, L., Oeuvres scientifiques (Editions du CNRS) 1978.

Herpin, A., Théorie du magnétisme (P.U.F.) 1968.

Chikazumi, S., Physics of Magnetism (John Wiley) 1964.

NYE, J. F., Propriétés physiques des cristaux, Traduction française (Dunod Paris) 1961.
Brown, W. F., Principes de Ferromagnétisme (Monographie Dunod) Paris 1970, traduction française de «Magnetostatic Principles in Ferromagnetism » (North-Holland Amsterdam).

Articles de revue ou mises au point; divers:

KitTEl, C., Physical theory of ferromagnetic domains, Rev. Mod. Phys. 21 (1949).

Brissonneau, P., Les domaines magnétiques, Rev. Phys. Appl. 9 (1974).

Vergne, R., Cotillard, J. C. et Porteseil, J. L., Quelques aspects statistiques des processus d'aimantation dans les corps ferromagnétiques, cas du déplacement d'une seule paroi de Bloch à $180^{\circ}$ dans un milieu monocristallin aléatoirement perturbé, Revue Phys. Appl. 16 (1981).

DORMANN, J. L., Le phénomène de superparamagnétisme, Revue Phys. Appl. 16 (1981).

Brown, W. F., Thermal Fluctuations of fine Ferromagnetic Particules, IEEE Trans. magnetism. Magn. 15 (1979) $\mathrm{n}^{\circ} 5$

Moskowitz, B. M. et Banerjee, S. K., Grain size units for pseudosingle domain behavior in magnetite. Implication for paleomagnetism, IEEE Trans. magnetism Magn. 15 (1979) $n^{\circ} 5$.

FULLER, M., On the grain size dependence of the behavior of fine magnetic particules in rocks, Geophys. Surveys 7 (1984).

Daly, L., Thèse de Doctorat d'état, Faculté des sciences de l'Université de Paris (1970).

\section{Références particulières}

[1] Fernandez, A., Cf. Bibliographie générale.

[2] GAY, N. C., Cf. Bibliographie générale.

[3] Tullis, T. E., Cf. Bibliographie générale.

[4] Debat, P., Sirieys, P., Deramond, J. et Soula, J. C., cf. Bibliographie générale.

[5] NÉEL, L., Oeuvres scientifiques, p. 205.

[6] Birss, R. R. et Hegarty, B. C., Brit. Appl. Phys. 17 (1966) 1241 à 1256.

[7] Molho, P., Porteseil, J. L., Souche, Y., GouZERH, J. et LEVY, J. C. S., Irreversible evolution in the topology of magnetic domains, à paraître dans J.M.M.M. Proc. of MMM 1986 (Baltimore).

[8] Molno, P., Thèse de Doctorat d'état, U.S.M.G. et I.N.P.G., Grenoble (1986).
[9] Chikazumi, S., Cf. Bibliographie générale.

[10] Néel, L., J. Phys. Soc. Jpn 17 (1962) suppl. B1.

[11] Rochette, P., Thèse de Doctorat de $3^{\text {c }}$ Cycle, I.R.I.G.M., Grenoble (1983).

[12] Guillet, J. P. et Almaric, R., Revue Phys. Appl. 14 (1979).

[13] Lliboutry, L., Thèse de Doctorat d'état, Faculté des Sciences de l'Université de Grenoble (1950) (Masson et Cie Eds) pp. 43, 44. Ce travail comporte de nombreuses références bibliographiques.

[14] Vergne, R., Ann. Geophys. 5 (1987) nº Sér. B. 